\title{
Trilepton signal for supersymmetry at the Fermilab Tevatron reexamined
}

\author{
Howard Baer, ${ }^{1}$ Manuel Drees, ${ }^{2}$ Frank Paige, ${ }^{3}$ Pamela Quintana, ${ }^{1}$ and Xerxes Tata ${ }^{4}$ \\ ${ }^{1}$ Department of Physics, Florida State University, Tallahassee, Florida 32306 \\ ${ }^{2}$ IFT, Universided Estadual Paulista, 01405-900 São Paulo, Brazil \\ ${ }^{3}$ Brookhaven National Laboratory, Upton, New York 11973 \\ ${ }^{4}$ Department of Physics and Astronomy, University of Hawaii, Honolulu, Hawaii 96822
}

(Received 3 June 1999; published 4 April 2000)

\begin{abstract}
Within a wide class of models, the CERN LEP2 lower limit of $95 \mathrm{GeV}$ on the chargino mass implies gluinos are heavier than $\sim 300 \mathrm{GeV}$. In this case electroweak $\widetilde{W}_{1} \bar{W}_{1}$ production and $\widetilde{W}_{1} \widetilde{Z}_{2}$ production are the dominant supersymmetry (SUSY) processes at the Fermilab Tevatron, and the extensively examined isolated trilepton signal from $\widetilde{W}_{1} \widetilde{Z}_{2}$ production assumes an even greater importance. We update our previous calculations of the SUSY reach of luminosity upgrades of the Fermilab Tevatron in this channel incorporating (i) decay matrix elements in the computation of the momenta of leptons from chargino and neutralino decays, (ii) the trilepton background from $W^{*} Z^{*}$ and $W^{*} \gamma^{*}$ production which, though neglected in previous analyses, turns out to be the dominant background, and finally, (iii) modified sets of cuts designed to reduce these new backgrounds and increase the range of model parameters for which the signal is observable. We show our improved projections for the reach for SUSY of both the Fermilab Main Injector and the proposed TeV33 upgrade. We also present opposite sign same flavor dilepton invariant mass distributions as well as the $p_{T}$ distributions of leptons in SUSY trilepton events, and comment upon how the inclusion of decay matrix elements impacts upon the Tevatron reach, as well as upon the extraction of neutralino masses.
\end{abstract}

PACS number(s): 14.80.Ly, 11.30.Pb, 13.85.Qk

\section{INTRODUCTION}

The minimal supergravity (MSUGRA) model is a well motivated framework [1] for exploring the experimental consequences of weak scale supersymmetry. In this model, it is assumed that supersymmetry (SUSY) breaking occurs in a "hidden sector" of the model, and is then communicated to the observable sector via gravitational-strength interactions. Motivated by the observed suppression of flavor changing neutral currents as well as by the near unification of gauge coupling constants at $M_{G U T} \simeq 2 \times 10^{16} \mathrm{GeV}$, one assumes a common mass $m_{0}$ for scalars and a common mass $m_{1 / 2}$ for gauginos at scale $Q=M_{G U T}$. In addition, the soft breaking trilinear $A$ terms are also unified to $A_{0}$ at $Q=M_{G U T}$. The soft SUSY breaking parameters, gauge and Yukawa couplings are then evolved from $Q=M_{G U T}$ to $Q \sim M_{\text {weak }}$ via renormalization group equations. The magnitude of the superpotential $\mu$ term is determined by requiring radiative breaking of electroweak symmetry; this latter constraint also effectively allows one to trade the bilinear $B$ parameter for the parameter $\tan \beta$. Thus, the entire spectrum of SUSY and Higgs particle masses (as well as all the couplings) is predicted in terms of standard model (SM) parameters augmented by the SUSY parameter set

$$
m_{0}, \quad m_{1 / 2}, \quad A_{0}, \quad \tan \beta \text { and } \operatorname{sgn}(\mu) .
$$

This model, along with several others, has been incorporated into the event generator ISAJET [2].

The negative results of sparticle searches in experiments at the Fermilab Tevatron and at CERN $e^{+} e^{-}$collider LEP have led to significant lower limits on gluino and chargino masses. In the MSUGRA framework and in other models with (real or apparent) gaugino mass unification, the LEP2 limit on chargino mass $m_{\tilde{W}_{1}}>95 \mathrm{GeV}[3]$ implies that gluinos and most squarks ought to have masses typically greater than about $300 \mathrm{GeV}$, so that strong sparticle production at the Tevatron is expected to be suppressed. Then, $\widetilde{W}_{1} \bar{W}_{1}$ production and $\widetilde{W}_{1} \widetilde{Z}_{2}$ production is expected ${ }^{1}$ to be the dominant sparticle production mechanism if sparticles are at all accessible. It has become increasingly clear that the trilepton signal from $p \bar{p} \rightarrow \widetilde{W}_{1} \widetilde{Z}_{2} X$ followed by $\widetilde{Z}_{2} \rightarrow l \bar{l} \widetilde{Z}_{1}$ and $\widetilde{W}_{1}$ $\rightarrow l \nu \widetilde{Z}_{1}(l=e, \mu)$ is one of the most promising discovery channels for supersymmetry at luminosity upgrades of the Fermilab Tevatron collider [4-10]. As an illustration, in Fig. 1 , we plot sparticle cross sections as a function of $m_{\tilde{g}}$ assuming five generations of degenerate squarks, for (a) $m_{\tilde{q}}=m_{\tilde{g}}$ and (b) $m_{\tilde{q}}=2 m_{\tilde{g}}$, assuming $\mu=+m_{\tilde{g}}$, and $\tan \beta=3$ (minimal supersymmetric standard model parameters with gaugino mass unification). The region to the left of the vertical line is excluded by the LEP2 chargino mass limit $m_{\widetilde{W}_{1}}$ $>95 \mathrm{GeV}$ [3]. It can be seen that electroweak production of charginos and neutralinos dominates over the strongly produced $\tilde{g} \tilde{g}, \tilde{g} \tilde{q}$, and $\tilde{q} \tilde{q}$ cross sections over essentially all of parameter space for which $|\mu| \gg M_{1}, M_{2}$. The cross section for $\widetilde{W}_{1} \widetilde{Z}_{1}$ is relatively suppressed because $\widetilde{Z}_{1}$ is dominantly a hypercharge gaugino and so couples to $W$ via its suppressed components, while the squark or gluino plus chargino or neutralino associated production reactions (summed over all

\footnotetext{
${ }^{1}$ This is not to imply that experiments at the Fermilab Tevatron ought not to search for gluinos and squarks. Direct search limits are important since our assumption about the relationships between gaugino masses could well prove to be incorrect.
} 

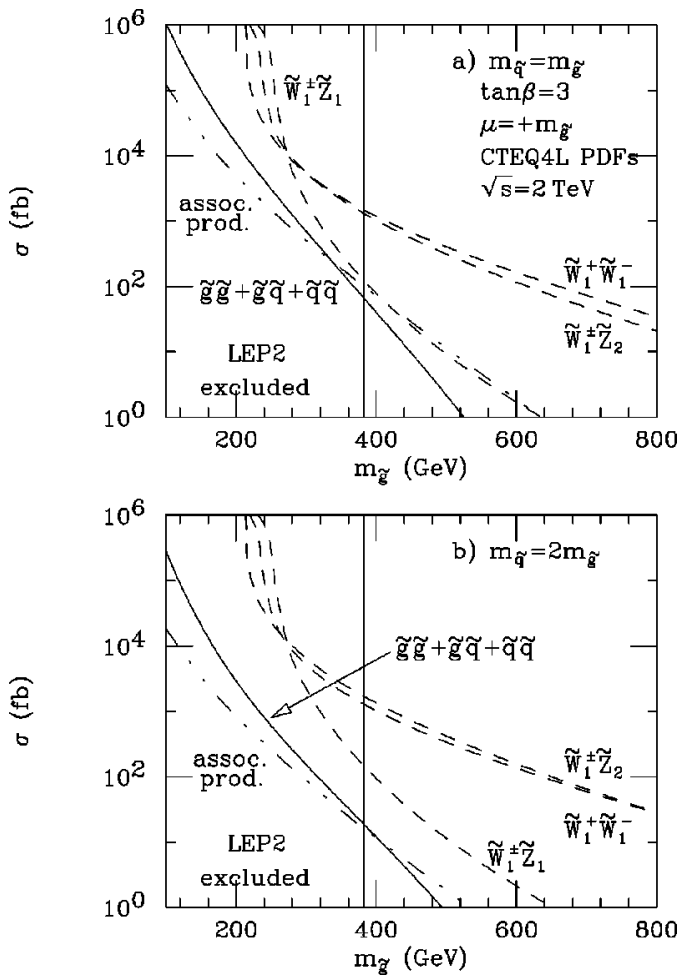

FIG. 1. Sparticle production cross sections as a function of $m_{\tilde{g}}$ for $\mu=+m_{\tilde{g}}$ and $\tan \beta=3$.

sparticle types and shown by dash-dot-dot curves) occur at smaller rates. These qualitative features hold for both frames shown. Similar results are shown in Fig. 2 for $\mu=-m_{g}^{\tilde{g}}$. In this case, for low values of $m_{\tilde{g}}$ with $m_{\tilde{q}} \simeq m_{\tilde{g}}$, the strong production cross sections can be dominant, but only in parameter space regions already excluded by LEP2. Since opposite sign dilepton or jet plus lepton signals from $\widetilde{W}_{1} \overline{\widetilde{W}}_{1}$ production suffer from large standard model backgrounds, many groups have focused on the clean trilepton signature from $\widetilde{W}_{1} \widetilde{Z}_{2}$ production for which the SM background is expected to be small: after suitable cuts, this mainly comes from $p \bar{p} \rightarrow W Z^{*}+X$ or $W \gamma^{*}+X$, where $W \rightarrow l \nu$ and $Z^{*}$ or $\gamma^{*}$ decay leptonically.

The Fermilab Tevatron reach in this channel has been extensively examined especially for low values of $\tan \beta$ where the effect of Yukawa interactions is negligible. In parts of parameter space of the MSUGRA model (including those favored by predicting a big bang relic abundance of lightest neutralinos in the cosmologically interesting range [11]), the reach is very large because neutralino leptonic decay rates can be enhanced owing in part to relatively light sleptons mediating the decay chain. However, in other regions of parameter space, these same decays can be suppressed by large negative interference terms between the $Z$ and slepton exchange graphs [12], and there is no reach even if charginos are just beyond the LEP bound.

If the MSUGRA parameter $\tan \beta$ is large, then $\tau$ and $b$ Yukawa couplings become non-negligible as well, and as a result, $\tilde{\tau}_{1}$ and $\tilde{b}_{1}$ can be significantly lighter than first and second generation sleptons and squarks. Consequently,
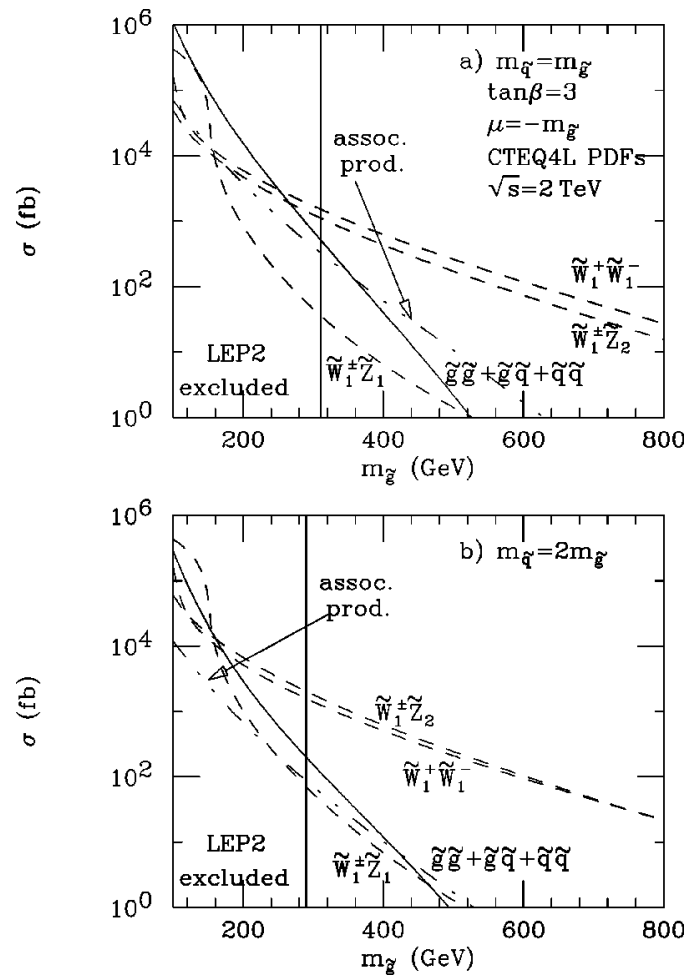

FIG. 2. Sparticle production cross sections as a function of $m_{\tilde{g}}$ for $\mu=-m_{\tilde{g}}$ and $\tan \beta=3$.

chargino and neutralino decays to $\tau$ leptons and $b$ quarks can be enhanced over decays to their first and second generation counterparts. If the decays $\widetilde{W}_{1} \rightarrow \tau \nu_{\tau} \widetilde{Z}_{1}$ and $\widetilde{Z}_{2} \rightarrow \tau \bar{\tau} \widetilde{Z}_{1}$ are strongly enhanced, trilepton events (where $e$ or $\mu$ are secondaries from $\tau$ decays) can still occur at a considerable rate, but then $e$ s and $\mu$ s will have a much softer energy distribution making the detection of the signal more challenging. Hence, unless hadronically decaying taus can be detected with high efficiency and purity, the Fermilab Tevatron reach for MSUGRA in general becomes more limited for high values of $\tan \beta[7]$.

Because of the importance of this channel, considerable attention was focused on optimizing cuts to maximize the Tevatron supersymmetry (SUSY) discovery potential during the recent Fermilab Tevatron "Workshop on SUSY/Higgs Particles at Run 2.' 'Barger, Kao, and Li [8] pointed out that using softer lepton $p_{T}$ cuts for SUSY trilepton events could improve the expected SUSY signal to background levels by a significant margin, particularly for scenarios with large values of $\tan \beta$. In addition $[9,13]$, the signal level could be increased significantly relative to background by allowing jetty events into the trilepton signal sample, and the greatest reach was shown to be obtained via this inclusive trilepton channel. The Fermilab Tevatron reach in MSUGRA parameter space was computed in Ref. [9] using soft cuts, and was found to have increased significantly beyond the results presented in Refs. [6,7], where harder lepton cuts (originally devised for the signal at low $\tan \beta$ ) and a jet veto were used. It has recently been pointed out [10] that these studies neglected contributions from $W Z$ production where the $Z$ boson was allowed to be off mass shell. These authors included 
TABLE I. Hard (HC1, HC2) and soft (SC1, SC2, SC3) cuts for Tevatron SUSY trilepton searches. See Sec. II for discussion.

\begin{tabular}{|c|c|c|c|c|c|}
\hline Cut & $\mathrm{HC} 1$ & $\mathrm{SC} 1$ & $\mathrm{SC} 2$ & $\mathrm{HC} 2$ & SC3 \\
\hline$p_{T}\left(l_{1}\right)$ & $>20 \mathrm{GeV}$ & $>11 \mathrm{GeV}$ & $>11 \mathrm{GeV}$ & $>20 \mathrm{GeV}$ & $>11 \mathrm{GeV}$ \\
\hline$p_{T}\left(l_{2}\right)$ & $>15 \mathrm{GeV}$ & $>7 \mathrm{GeV}$ & $>7 \mathrm{GeV}$ & $>15 \mathrm{GeV}$ & $>7 \mathrm{GeV}$ \\
\hline$p_{T}\left(l_{3}\right)$ & $>10 \mathrm{GeV}$ & $>5 \mathrm{GeV}$ & $>5 \mathrm{GeV}$ & $>10 \mathrm{GeV}$ & $>5 \mathrm{GeV}$ \\
\hline$\left|\eta\left(l_{1,2 / 3}\right)\right|$ & $<2.5$ & $<1.0,2.0$ & $<1.0,2.0$ & $<1.0,2.0$ & $<1.0,2.0$ \\
\hline$I S O_{\Delta R=0.4}$ & $<2 \mathrm{GeV}$ & $<2 \mathrm{GeV}$ & $<2 \mathrm{GeV}$ & $<2 \mathrm{GeV}$ & $<2 \mathrm{GeV}$ \\
\hline $\boldsymbol{E}_{T}$ & $>25 \mathrm{GeV}$ & $>25 \mathrm{GeV}$ & $>25 \mathrm{GeV}$ & $>25 \mathrm{GeV}$ & $>25 \mathrm{GeV}$ \\
\hline$Z$-veto & $83-99 \mathrm{GeV}$ & $81-101 \mathrm{GeV}$ & $<81 \mathrm{GeV}$ & $<81 \mathrm{GeV}$ & \\
\hline$N(j)$ & 0 & & & & \\
\hline$m(l \bar{l})$ & & & $>20 \mathrm{GeV}$ & $>12 \mathrm{GeV}$ & \\
\hline$m_{T}\left(l, \boldsymbol{E}_{T}\right)$-veto & & & $60-85 \mathrm{GeV}$ & $60-85 \mathrm{GeV}$ & \\
\hline OS/SF veto & No & No & No & No & Yes \\
\hline
\end{tabular}

backgrounds from $W Z^{*}$ production using PYTHIA, and looked to optimize cuts for SUSY trilepton signals over background throughout the MSUGRA model parameter space. In fact, in much of parameter space, they found hard lepton cuts were optimal since they could remove more of the $W Z^{*}$ background that was incompletely removed by a $Z$-boson mass veto.

Recently, the event generator ISAJET ( $v$ 7.44) has been upgraded to include decay matrix elements for chargino, neutralino, and gluino three-body decays. In previous versions, while the computation of branching fractions had included these matrix elements, just phase space was used in the event generation to determine the energy and momentum distributions of their decay products. In practice, because various kinematic cuts are placed on each of the three signal leptons in order to extract the signal from the background, the resultant signal rates can in fact depend on the momentum and energy distributions of the decay leptons. This neglect of the matrix element becomes an especially poor approximation when the mass of the gauge boson mediating the decay approaches that of the parent neutralino or chargino. Moreover, as has recently been pointed out by Nojiri and Yamada [14], distributions in dilepton invariant mass $m\left(l^{+} l^{-}\right)$can be sensitive (due to matrix element effects) to SUSY particle masses and mixings. These can affect not only the overall $m\left(l^{+} l^{-}\right)$distribution shape, but also the determination of the distribution endpoint, which yields an important measure of the mass difference $m_{\tilde{Z}_{2}}-m_{\tilde{Z}_{1}}$.

In light of these various developments, and the importance of the trilepton signal to SUSY searches at the Fermilab Tevatron, we felt it was worthwhile to update our calculations for the reach of Fermilab Tevatron upgrades for the MSUGRA model via the trilepton channel. We include decay matrix elements for chargino and neutralino decays in the generation of SUSY events. We also perform an exact lowest order calculation of the background process $p \bar{p} \rightarrow l \nu$ $+l^{\prime} \bar{l}^{\prime} X$, including both $W^{*} \gamma^{*}$ and $W^{*} Z^{*}$ contributions. The $W^{*} \gamma^{*}$ source gives background rates beyond those considered in Ref. [10]. We show reach results for both soft and hard lepton cuts, augmented by some additional cuts designed to eliminate much of this new background.

In Sec. II, we describe our background calculations, and list the improved soft and hard cuts that we use to reduce these backgrounds relative to the SUSY signals. In Sec. III, we describe in some detail our inclusion of decay matrix elements in ISAJET, and examine some distributions which reflect their inclusion. In Sec. IV, we perform five case studies of the trilepton signal for each set of selection cuts introduced in Sec. II and obtain the best one for the extraction of the signal. We also study the effect of the matrix element on the total signal as well as on various distributions. In Sec. V we present the results of our updated calculations of the SUSY trilepton reach in the $m_{0}$ vs $m_{1 / 2}$ plane. We conclude in Sec. VI with a summary of our results and some general remarks.

\section{BACKGROUNDS AND SELECTION CUTS}

We use ISAJET 7.44 to generate events in the MSUGRA model parameter space and to generate most of the SM backgrounds. We use the toy detector simulation package ISAPLT, assuming calorimetry between $-4<|\eta|<4$, with an array of calorimeter cells of size $\Delta \eta \times \Delta \phi=0.1 \times 0.262$. We take the electromagnetic energy resolution to be $0.15 / \sqrt{E}$ and the hadronic calorimeter resolution to be $0.7 / \sqrt{E}(E$ in $\mathrm{GeV})$. Calorimeter cells are coalesced in towers of $\Delta R=0.7$ using the jet finding algorithm GETJET. Hadronic clusters with $E_{T}(j)>15 \mathrm{GeV}$ are called jets. Leptons ( $e$ s or $\mu \mathrm{s}$ ) with $p_{T}$ of $5 \mathrm{GeV}$ or more are considered to be isolated if the hadronic $E_{T}$ in a cone about the lepton of $\Delta R=0.4$ is less than 2 $\mathrm{GeV}$.

We have examined the signals and backgrounds using five sets of acceptance cuts. A relatively hard set of cuts chosen for the study of the clean trilepton reach for low values of $\tan \beta$ is taken from Ref. [5] (note, however, that the lepton isolation criterion that we use here differs slightly from Ref. [5]). These cuts are listed in column 2 of Table I, and labeled HC1. The Collider Detector at Fermilab [15] and D0 [16] Collaborations and Mrenna et al. [17] have used relatively softer cuts in their analyses. These soft cuts were advocated 
TABLE II. Standard model backgrounds (fb) to the Tevatron SUSY trilepton signal for the hard as well as for the soft cuts listed in Table I.

\begin{tabular}{lccccc}
\hline \hline BG & HC1 & SC1 & SC2 & HC2 & SC3 \\
\hline$W Z(Z \rightarrow \tau \bar{\tau})$ & $0.175 \pm 0.005$ & $0.40 \pm 0.01$ & $0.28 \pm 0.01$ & $0.16 \pm 0.004$ & $0.106 \pm 0.004$ \\
$W^{*} Z^{*}, W^{*} \gamma^{*} \rightarrow l l \bar{l}$ & $1.70 \pm 0.05$ & $22.0 \pm 2.0$ & $0.21 \pm 0.02$ & $0.09 \pm 0.01$ & 0 \\
$W^{*} Z^{*}, W^{*} \gamma^{*} \rightarrow l l^{\prime} \bar{l}^{\prime}$ & $2.43 \pm 0.04$ & $14.6 \pm 0.4$ & $0.48 \pm 0.02$ & $0.22 \pm 0.003$ & 0 \\
$t \bar{t}$ & $<0.003$ & $0.14 \pm 0.006$ & $0.04 \pm 0.01$ & $0.003 \pm 0.003$ & $<0.003$ \\
$Z^{*} Z^{*}$ & $0.008 \pm 0.001$ & $0.07 \pm 0.002$ & $0.04 \pm 0.001$ & $0.02 \pm 0.001$ & $0.010 \pm 0.001$ \\
Total & 4.31 & 37.2 & 1.05 & 0.49 & 0.116 \\
$5 \sigma / 5 e v \cdot\left(2 \mathrm{fb}^{-1}\right)$ & 7.3 & 21.6 & 3.62 & 2.5 & 2.5 \\
$5 \sigma\left(25 \mathrm{fb}^{-1}\right)$ & 2.1 & 6.1 & 1.02 & 0.70 & 0.34 \\
$3 \sigma\left(25 \mathrm{fb}^{-1}\right)$ & 1.24 & 3.7 & 0.61 & 0.42 & 0.20 \\
\hline \hline
\end{tabular}

in Refs. $[8,9,13]$ as being more effective in eliciting signal from background, especially for large $\tan \beta$, where many of the signal leptons arise as secondaries from $\tau$ decay, and are quite soft. These cuts are listed in column 3 of Table I, and labeled SC1. Note that unlike for $\mathrm{HC1}$, a jet veto is not imposed, so that the signal will be inclusive, containing both clean and jetty trilepton events. We list in column 4 the augmented soft cuts that allow significant suppression of the $W^{*} \gamma^{*}$ and $W^{*} Z^{*}$ backgrounds; these are labeled SC2. In column 5, we modify the SC2 cuts imposing hard lepton $p_{T}$ requirements, and denote this new set by $\mathrm{HC} 2$. For large values of $\tan \beta$ where $\widetilde{Z}_{2} \rightarrow \tau \bar{\tau} \widetilde{Z}_{1}$ dominates the decay of $\widetilde{Z}_{2}$, trilepton events arise when both $\tau$ s decay leptonically, with a third lepton coming from chargino decay. In this case, the opposite sign (OS) dilepton pair in the trilepton event does not have the same flavor (SF) in a quarter of the signal events, regardless of the origin of the third lepton. Since these secondary leptons are soft, we use cuts $\mathrm{SC} 1$ but veto events with OS/SF pairs to reject the $Z^{*}$ and $\gamma^{*}$ backgrounds; this cut set (which is introduced to pick up the large $\tan \beta$ signal) is labeled by SC3.

The dominant SM backgrounds are listed in Table II for the five sets of cuts. It has recently been pointed out [10] that $W Z$ production, where $W \rightarrow l \nu$ and an off-shell $Z^{*} \rightarrow l \bar{l}$ is a major background to the SUSY trilepton signal. Similarly, $W \gamma^{*}$ can also lead to significant rates for trilepton backgrounds. ${ }^{2}$ We have used the program MADGRAPH or HELAS [18] to compute the complete lowest order squared matrix element for the process $q \bar{q}^{\prime} \rightarrow e^{+} \nu_{e} \mu^{+} \mu^{-}$(we subsequently learned that this background was also computed by Campbell and Ellis, Ref. [19]). The ten contributing Feynman diagrams are shown in Fig. 3, and include contributions from $W^{*} Z^{*}$ and $W^{*} \gamma^{*}$ production, plus some other contributions. (Throughout this paper, a star on a particle indicates

\footnotetext{
${ }^{2}$ The importance of this contribution to the background was first pointed out in Ref. [17], which also contains an estimate of its size. Our results differ significantly from this estimate, especially for small dilepton invariant masses, where our calculation gives much higher rates, due to the $\gamma$ pole.
}

that it could be either real or virtual.) Similarly, we have computed the $q \bar{q}^{\prime} \rightarrow e^{+} \nu_{e} e^{+} e^{-}$background, which includes 20 diagrams. We have constructed parton-level Monte Carlo programs to then estimate these backgrounds. We use ISAJET to calculate the $t \bar{t}$ and $W Z(Z \rightarrow \tau \bar{\tau})$ backgrounds. In addition, we use ISAJET to calculate backgrounds from $Z Z$ production. For this latter calculation, we smear each $Z$ decay to $e \bar{e}$ or $\mu \bar{\mu}$ by a Breit-Wigner distribution to simulate the effect of the off-shell $Z$ contribution. This cross section is much larger with $\mathrm{HC} 2$ cuts than $\mathrm{HC} 1$ cuts because events

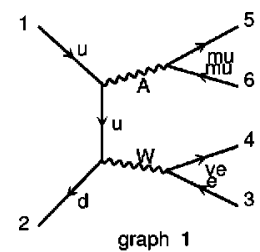

graph 1

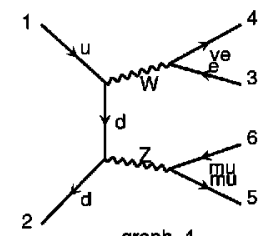

graph 4
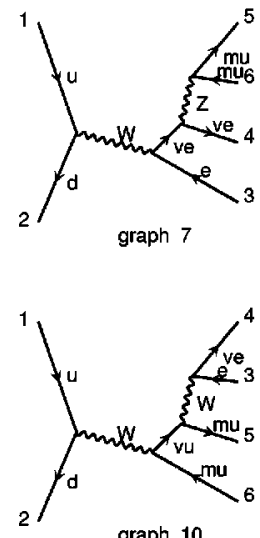

graph 10

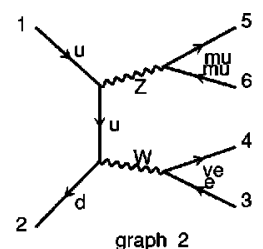

graph 2

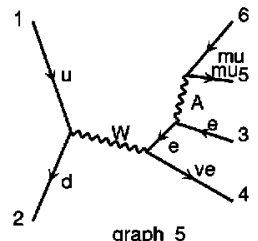

graph 5

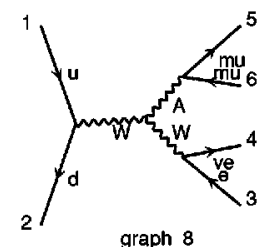

graph 8

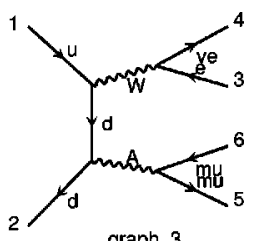

graph 3

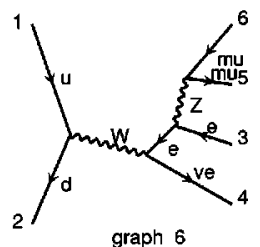

graph 6

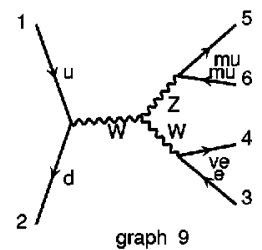

graph 9
FIG. 3. Feynman diagrams contributing to $W^{*} \gamma^{*}, W^{*} Z^{*}$ background. 

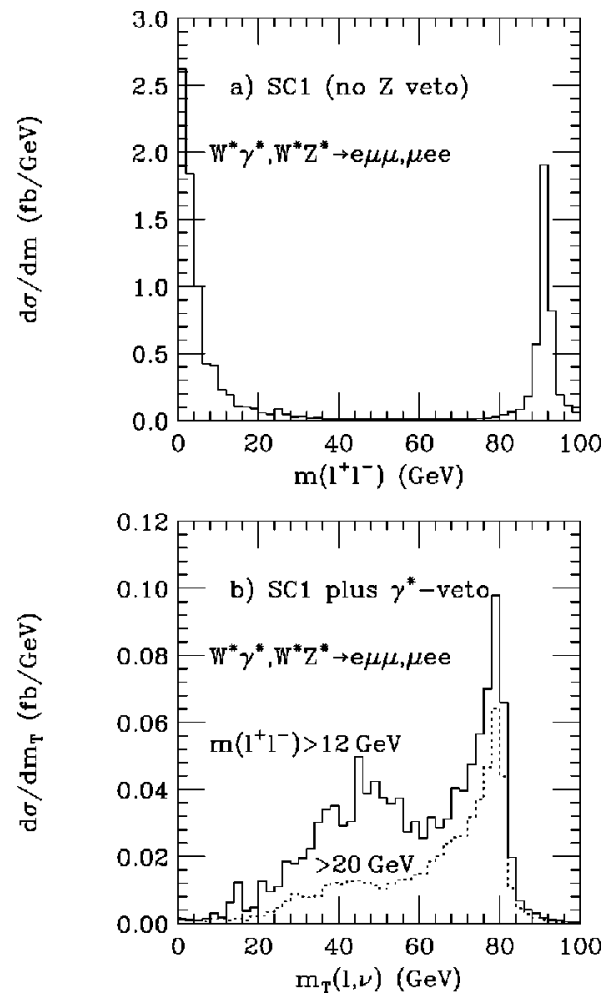

FIG. 4. (a) Distribution of same-flavor, opposite sign dilepton mass from $W^{*} \gamma^{*}, W^{*} Z^{*} \rightarrow e \mu \mu$, $\mu e e$ background after cuts $\mathrm{SC} 1$, but with the Z-mass veto removed. In (b), we show the distribution in transverse mass from the same background with cuts SC1, including the $Z$ and $\gamma$ veto.

from $Z^{*} \rightarrow l l$ while $Z \rightarrow \tau \tau$ (where just one of the two taus decay leptonically) would be mostly removed by the jet veto involved in $\mathrm{HC} 1$, but would likely pass the $\mathrm{HC} 2$ inclusive cuts.

From Table II, it can be seen that using HC1 or SC1 cuts, there is a very large background contribution from the $l \nu l^{\prime} \bar{l}^{\prime}$ source (denoted by $W^{*} Z^{*}$ or $W^{*} \gamma^{*}$ ). We plot in Fig. 4(a) the dilepton mass distribution for same flavor-opposite sign dilepton pairs after imposing the $\mathrm{SC} 1$ cuts, except for the $Z$ veto. In performing our parton level Monte Carlo for $l \nu l^{\prime} \bar{l}^{\prime}$ production, we cut off $l^{\prime} \bar{l}^{\prime}$ masses below $1 \mathrm{GeV}$ to avoid the singularity from the $\gamma$ propagator. It is also crucial to integrate over the entire range of invariant mass values for the virtual $W$ contribution since we find a large background contribution coming from $W^{*}$, s with invariant mass in the range of $30-60 \mathrm{GeV}$.

There is a large contribution [19] to the $3 l$ background from the $Z$ resonance, much of which is effectively removed by the $Z$ mass veto cut. Here, we expand the $Z$ mass veto cut to include all events with $m(l \bar{l})>81 \mathrm{GeV}$, which vetos far off-shell $\gamma^{*}$ or $Z^{*}$ decays (an $m\left(l^{+} l^{-}\right)>81 \mathrm{GeV}$ cut was suggested previously in Ref. [10]). An even larger contribution comes from the phase-space region where the photon gets close to its mass shell: a " $\gamma^{*}$ veto," must be imposed to reduce the large $W^{*} \gamma^{*}$ contribution at lower dilepton mass values. This cut should also remove background from charmonium and bottomonium decays and from $b$ decays [10]. In
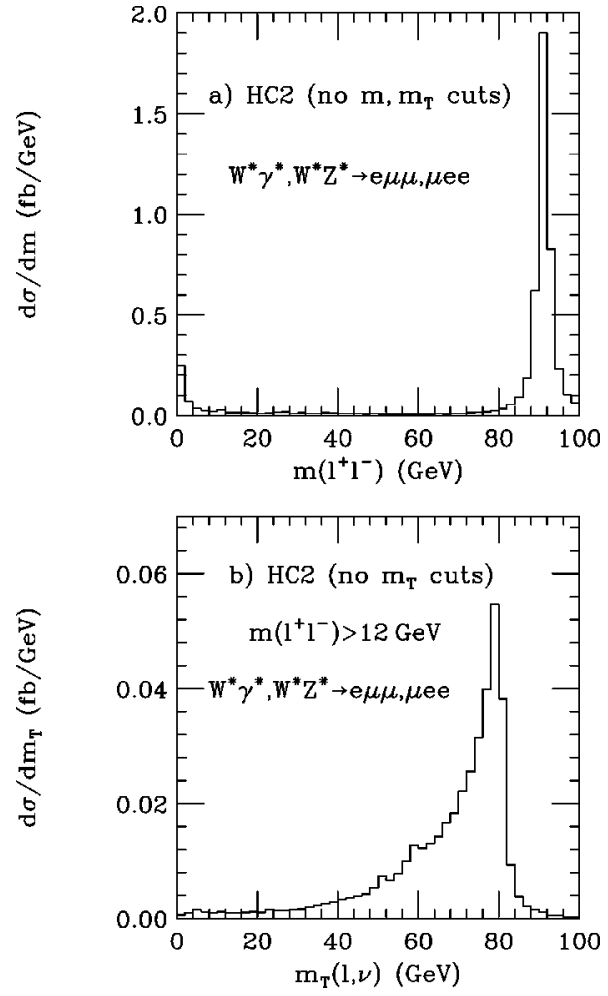

FIG. 5. (a) Distribution of same-flavor, opposite sign dilepton mass from $W^{*} \gamma^{*}, W^{*} Z^{*} \rightarrow e \mu \mu$, $\mu e e$ background after cuts HC2, but with the $m\left(l^{+} l^{-}\right)$and $m_{T}$ cuts removed. In (b), we show the distribution in transverse mass from the same background with cuts HC2, including the $Z$ and $\gamma$ veto, but without the $m_{T}$ cut.

Fig. 4(b), we show the distribution in $m_{T}(l, \nu)$ after using SC1 plus a $\gamma^{*}$ mass veto of either 12 or $20 \mathrm{GeV}$. Imposing the $m\left(l^{+} l^{-}\right)>12 \mathrm{GeV}$ cut leaves a characteristic $W$-boson transverse mass distribution, but with a distinctive bulge around $m_{T}(l, \nu) \sim 40-60 \mathrm{GeV}$ due to a large rate for far off mass shell $W^{*}$ production. The low mass $W^{*}$ 's are produced in association with very low mass $\gamma^{*}$ 's. Imposing instead $m\left(l^{+} l^{-}\right)>20 \mathrm{GeV}$ removes much of the off-shell $W$ bulge and leaves a more typical distribution in transverse mass. Much of the remaining background can be eliminated at some cost to signal by vetoing events with $60<m_{T}(l, \nu)$ $<85 \mathrm{GeV}$. Insertion of this complete background process into an event generator with QCD radiation and detector simulation will broaden the $m_{T}$ peak somewhat, increasing the background rate; however, some of this background rate may be decreased as well due for instance to nonisolated leptons. The cuts SC1 augmented by the $20 \mathrm{GeV}$ " $\gamma^{*}$ ", together with the expanded $Z$ veto and the $W$ transverse mass veto form our set of cuts SC2. We also examine a set of cuts $\mathrm{HC} 2$ which is $\mathrm{SC} 1$, but with increased $p_{T}$ requirements on each of the three leptons together with the $Z, \gamma^{*}$ and transverse mass vetos. Corresponding distributions in $m\left(l^{+} l^{-}\right)$ and $m_{T}(l, \nu)$ are shown in Fig. 5. From this figure, it is clear that for hard lepton $p_{T}$ cuts, a " $\gamma^{*}$ veto" $m\left(l^{+} l^{-}\right)$ $>12 \mathrm{GeV}$ is sufficient. Finally, we examine the cut set SC1, but augmented instead by a veto on OS/SF dilepton pairs, denoted by SC3. There is, of course, no background from $W^{*} Z^{*}$ and $W^{*} \gamma^{*}$ events and, as expected, the dominant 
remaining background from $W Z, Z \rightarrow \tau \tau$ events drops to about a fourth.

We also ran $Z+$ jets and $W+$ jets background jobs. No events from these two sources passed any of the sets of cuts out of $5 \times 10^{5}$ and $10^{6}$ events generated, respectively. These correspond to one event levels of less than 0.3 and $4 \mathrm{fb}$, respectively. In runs of $10^{8} \mathrm{~W}+$ jets events with somewhat different cuts, some $3 l$ events could be generated leading to sizeable backgrounds; these sources always had $b \rightarrow c l \nu$ followed by $c \rightarrow s l \nu$, so that these sources of background could be removed (without any appreciable loss of signal) by imposing an angular separation cut between the isolated leptons, giving a background consistent with zero. Finally, we list in Table II the total background cross section as well as the minimum signal levels for a $5 \sigma$ excess for integrated luminosity of 2 and $25 \mathrm{fb}^{-1}$ as well as the minimum for a " $3 \sigma$ observation" with $25 \mathrm{fb}^{-1}$. At Run II with $2 \mathrm{fb}^{-1}$ integrated luminosity, we expect about two (one) events per experiment using cuts $\mathrm{SC} 2$ ( $\mathrm{HC} 2)$, for which the background cross section is $\sim 1 \mathrm{fb}(\sim 0.5 \mathrm{fb})$. Thus, about seven signal events will be necessary to establish a $5 \sigma$ effect at Run II for $\mathrm{SC} 2$ cuts. We do not attempt to quote the increased significance that might be possible by combining the event samples from the two experiments. On the other hand, we do not attempt to model experimental efficiencies, either.

\section{DECAY MATRIX ELEMENT EFFECTS}

The event generator ISAJET 7.44 has recently been upgraded to include the effects of sparticle decay squared matrix elements on the distribution for any gluino or gaugino to decay to another gaugino plus a fermion-antifermion pair. Other matrix elements may be incorporated in the future. Spin correlations are not yet included in ISAJET, so the SUSY particles are effectively unpolarized, but these effects are probably less important. The procedure used is as follows:

(1) We begin by computing a general form for the decay amplitude for the process $\widetilde{A} \rightarrow \widetilde{B}+f+\bar{f}^{\prime}$, where $\widetilde{A}$ and $\widetilde{B}$ are gauginos or gluinos, and $f$ and $\bar{f}^{\prime}$ are SM fermions. We construct general amplitudes, i.e., amplitudes with arbitrary couplings consistent with the most general Lorentz structure, for decays via intermediate vector bosons, sfermions, antisfermions, scalar and pseudoscalar particles. The squared amplitudes including interference terms are all preprogrammed functions in ISAJET.

(2) When each decay branching fraction is calculated in ISAJET, the masses and types of each exchanged particle are saved along with the in general complex coefficients needed to specify the vertices. In our decay calculations, we include all third generation mixing effects, Yukawa couplings and tree level decay Feynman graphs [7].

(3) When any type of three body decay is generated, hitor-miss Monte Carlo is used to generate an appropriate kinematical set of decay product four-vectors. The maximum of the decay integrand, which is needed for this calculation, is calculated the first time via Monte Carlo integration. This result is saved and updated as better maxima are found.

In addition, ISAJET includes a calculation of partial widths of particle decays into left or right-handed taus indepen-

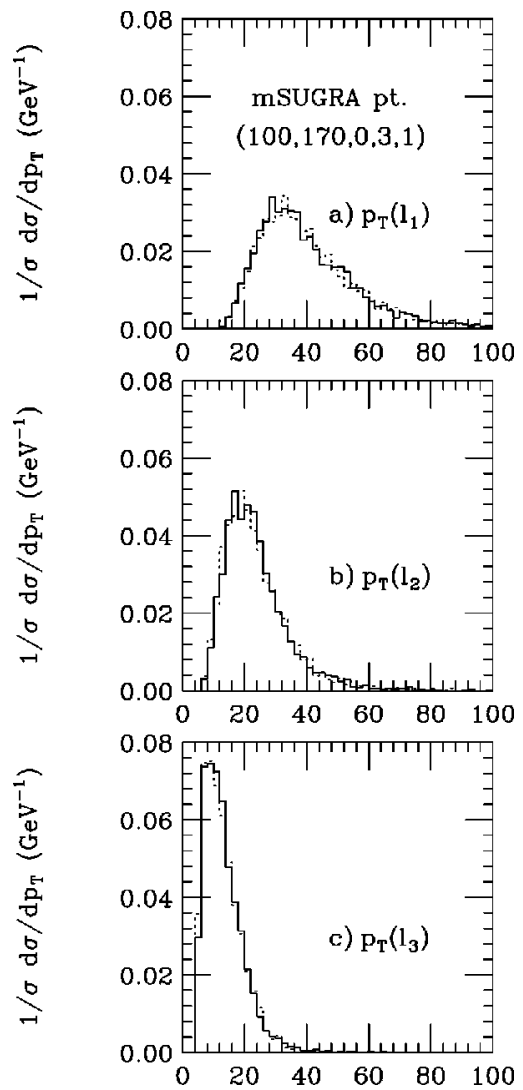

FIG. 6. Normalized isolated lepton $p_{T}$ distributions for the (a) highest, (b) second highest and (c) lowest $p_{T}$ lepton in trilepton events for the listed MSUGRA point (case A) after inclusive soft cuts SC1 listed in Table I. The dashed histogram denotes the case of phase space decays, while the solid histogram denotes the case with exact three-body decay matrix elements.

dently. Taus are then decayed appropriately according to their respective squared matrix elements. Spin correlations are neglected. For the case of sparticles decaying into two taus (such as $\widetilde{Z}_{2} \rightarrow \widetilde{Z}_{1} \tau \bar{\tau}$ ), an average $\tau$ polarization is used. Thus the effect of tau polarization (which plays an important role for signals involving hadronically decaying taus $[20,7,10])$ is at least approximately included. QCD corrections to both the signal and the background are neglected in our analysis.

The efficiency with which leptons from $\widetilde{W}_{1}$ and $\widetilde{Z}_{2}$ decays pass our cuts depends directly on their transverse momentum spectra. These are shown for three MSUGRA cases in Figs. 6,7 , and 8 , where we compare phase space distributions (dashed histograms) with predictions with the matrix element included (solid histograms) after the soft cuts SC1. Since our purpose is to illustrate the effect of the matrix elements for $\widetilde{W}_{1}$ and $\widetilde{Z}_{2}$ decays, these distributions are shown for just $\widetilde{W}_{1} \widetilde{Z}_{2}$ production, and not for the case where all SUSY processes are included. In Fig. 6 we illustrate these for case A with $\left[m_{0}, m_{1 / 2}, A_{0}, \tan \beta, \operatorname{sgn}(\mu)\right]=(100,170,0,3,1)$ (where dimensionful quantities are in $\mathrm{GeV}$ units). In this case, $m_{\tilde{Z}_{2}}=117 \mathrm{GeV}, m_{\tilde{Z}_{1}}=62 \mathrm{GeV}$ and $m_{\tilde{l}_{R}}=126 \mathrm{GeV}$, so that slepton exchange dominates leptonic $\widetilde{Z}_{2}$ decays. Be- 


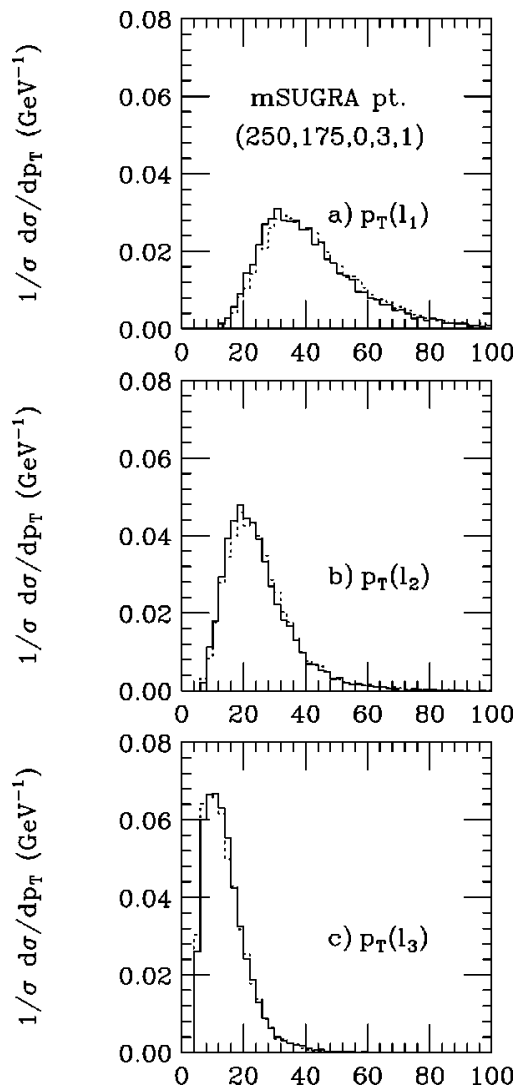

FIG. 7. Same as Fig. 6, except with MSUGRA parameters for case B.

cause the $\widetilde{l}_{R}$ mass is so close to $m_{\tilde{Z}_{2}}$ it would seem reasonable to expect that the inclusion of the matrix element would tend to enhance the rate for configurations where the third lepton is very soft (since this brings the intermediate slepton closest to its mass shell). We see, however, that the matrix elements predict very similar $p_{T}$ spectra for all three leptons compared to just phase space, so that in this case the phase space approximation works surprisingly well. A closer look at the squared matrix element for the "slepton-mediated $\widetilde{Z}_{2}$ decay" reveals that it actually vanishes in the limit that the momentum of either of its daughter leptons goes to zero, completely nullifying the enhancement expected from the propagator. For case B in Fig. 7 , with $\left[m_{0}, m_{1 / 2}, A_{0}\right.$, $\tan \beta, \operatorname{sgn}(\mu)]=(250,175,0,3,1)$, there is a large negative interference between $Z$ and slepton mediated decay graphs. In this case, for the two highest $p_{T}$ decay leptons, the results with exact decay matrix elements give a slightly softer $p_{T}$ spectrum, while that of the third lepton is somewhat harder. Figure 8 shows results for case $\mathrm{C}$ where we choose $\left[m_{0}, m_{1 / 2}, A_{0}, \tan \beta, \operatorname{sgn}(\mu)\right]$ $=(500,200,0,3,-1)$ for which $m_{\tilde{Z}_{2}}=173 \mathrm{GeV}, m_{\tilde{Z}_{1}}$ $=86 \mathrm{GeV}$ and $m_{\tilde{l}_{R}}=507 \mathrm{GeV}$. The parameters are chosen such that $\widetilde{Z}_{2}$ can decay through a nearly on-shell $Z$ boson; the leptons from $\widetilde{Z}_{2}$ decays are then usually quite energetic. As a result, the inclusion of the matrix element results in a considerably harder $p_{T}$ distribution for the three leptons relative to expectation based on phase space alone.

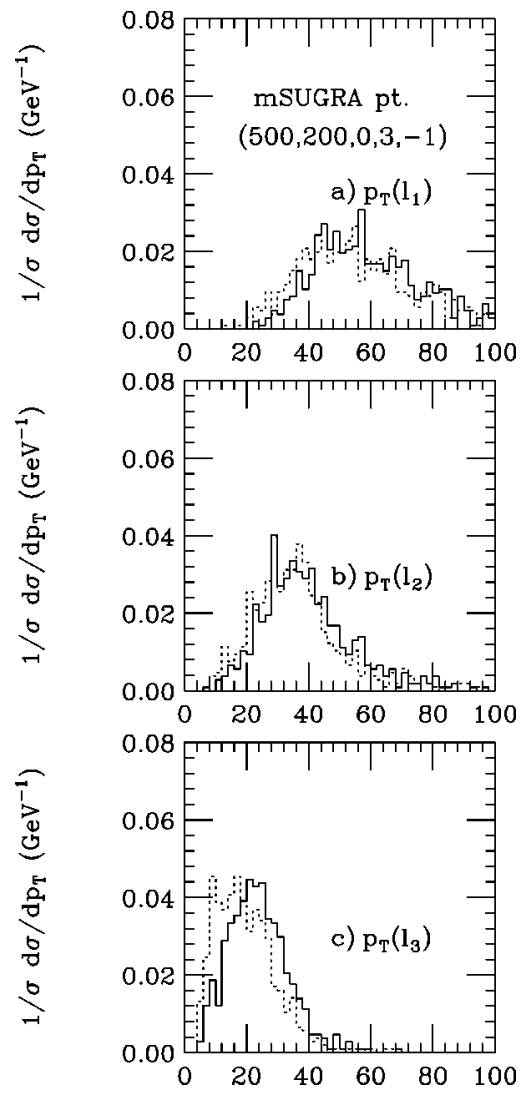

FIG. 8. Same as Fig. 6, except with MSUGRA parameters for case $\mathrm{C}$.

In Fig. 9, we show the mass distribution of opposite sign (OS) same flavor dileptons produced in $\widetilde{W}_{1} \widetilde{Z}_{2}$ events for the same MSUGRA point as in Fig. 6 (case A), where virtual slepton exchange dominates the $\widetilde{Z}_{2} \rightarrow \widetilde{Z}_{1} l \bar{l}$ decay. Again, the phase space distribution is denoted by dashes, while the exact results are solid. We use the SC1 cuts described in Sec. II in this figure [except for removing the $m(l \bar{l})$ cut around

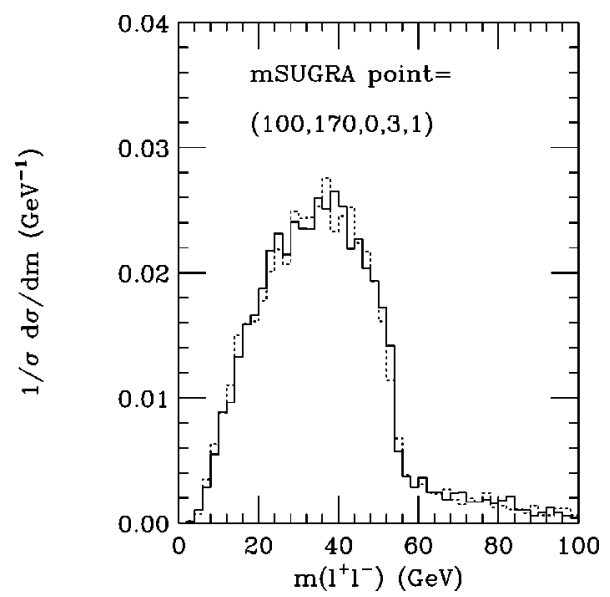

FIG. 9. Normalized opposite sign, same-flavor dilepton invariant mass distribution after inclusive soft cuts SC1 (other than the $Z$ veto) for the listed MSUGRA point, case A. We show the result using only phase space for the decay matrix element (dashes), and the result using the exact decay squared matrix element (solid). 


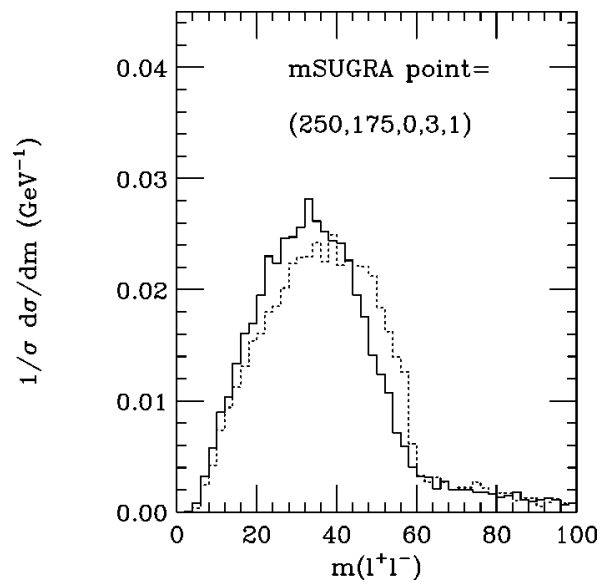

FIG. 10. The same as Fig. 9 except for case B where the model parameters are as listed.

$\left.M_{Z}\right]$, but include no background, and normalize to unity. For this point, the invariant mass of $l^{+} l^{-}$pairs from $\widetilde{Z}_{2}$ decays is bounded by $m_{\tilde{Z}_{2}}-m_{\tilde{Z}_{1}}=55.3 \mathrm{GeV}$; the few events at larger invariant masses arise from the twofold ambiguity present when all three leptons are the same flavor. For this case, there is hardly any shift in invariant mass between the two cases.

In Fig. 10, we show the $m(l \bar{l})$ distribution for case B also shown in Fig. 7 where the intermediate sleptons are quite heavy. In this case, there is a distinct shift of the distribution towards lower invariant masses when the decay matrix element is included. This arises from a cancellation between $Z$ and slepton mediated decay graphs which actually suppress the invariant mass distribution near its kinematic limit, as first noted by Nojiri and Yamada [14]. This situation can lead to potentially greater uncertainties in measuring the $\widetilde{Z}_{2}-\widetilde{Z}_{1}$ mass difference.

Finally, in Fig. 11, we show the $m(l \bar{l})$ distribution for case $\mathrm{C}$ where sleptons are very heavy, but the intermediate $Z$ boson in the decay process can be nearly on mass shell. As might be expected, the $Z$ pole pulls the dilepton invariant mass towards $M_{Z}$, and clearly illustrates the inadequacy of

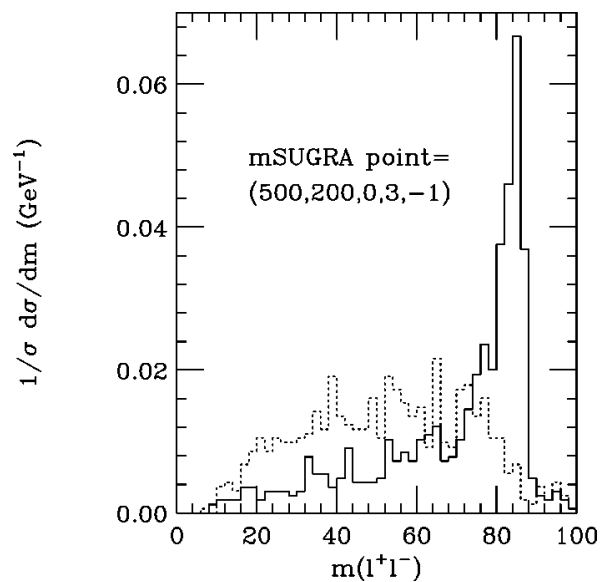

FIG. 11. The same as Fig. 9 except for case $\mathrm{C}$ where the model parameters are as listed.
TABLE III. Parameter space choices, sparticle masses, and total signal cross sections for the five case studies in Sec. IV. We also list the fractional contribution to the signal from various subprocesses. We take $m_{t}=175 \mathrm{GeV}$.

\begin{tabular}{|c|c|c|c|c|c|}
\hline Case & (1) & (2) & (3) & (4) & (5) \\
\hline$m_{0}$ & 100 & 140 & 200 & 250 & 150 \\
\hline$m_{1 / 2}$ & 200 & 175 & 140 & 150 & 300 \\
\hline$A_{0}$ & 0 & 0 & -500 & -600 & 0 \\
\hline $\tan \beta$ & 3 & 35 & 35 & 3 & 30 \\
\hline$m_{H_{u}}, m_{H_{d}}$ & & & & & 500,500 \\
\hline$m_{\tilde{g}}$ & 508 & 455 & 375 & 403 & 734 \\
\hline$m_{\tilde{q}}$ & 450 & 410 & 370 & 415 & 650 \\
\hline$m_{\tilde{t}_{1}}$ & 306 & 297 & 153 & 134 & 440 \\
\hline$m_{\tilde{b}_{1}}$ & 418 & 329 & 213 & 346 & 566 \\
\hline$m_{\tilde{W}_{1}}$ & 141 & 126 & 106 & 109 & 100 \\
\hline$m_{\tilde{Z}_{1}}$ & 76 & 69 & 56 & 57 & 80 \\
\hline$m_{\tilde{Z}_{2}}$ & 143 & 127 & 107 & 111 & 124 \\
\hline$m_{\tilde{Z}_{3}}$ & 316 & 252 & 296 & 373 & 141 \\
\hline$m_{\tilde{l}_{R}}$ & 132 & 162 & 212 & 260 & 195 \\
\hline$m_{\tilde{l}_{L}}^{\kappa}$ & 180 & 194 & 229 & 275 & 266 \\
\hline$m_{\tilde{\tau}_{1}}^{L}$ & 131 & 104 & 88 & 257 & 132 \\
\hline$m_{h}$ & 99 & 110 & 112 & 104 & 115 \\
\hline$\mu$ & 312 & 241 & 286 & 369 & -110 \\
\hline$\sigma_{\text {tot }}(\mathrm{fb})$ & 404 & 653 & 2712 & 3692 & 1393 \\
\hline$\tilde{g}, \tilde{q}(\%)$ & 4.3 & 6.6 & 50.4 & 66.2 & 0.01 \\
\hline$\tilde{g} \tilde{\chi}, \tilde{q} \tilde{\chi}(\%)$ & 2.4 & 3.6 & 2.9 & 1.2 & 0.01 \\
\hline$\tilde{\chi} \tilde{\chi}(\%)$ & 85.0 & 85 & 45.7 & 32.6 & 99.5 \\
\hline$\widetilde{l} \widetilde{l}(\%)$ & 8.3 & 4.7 & 1.0 & 0.04 & 0.4 \\
\hline$\tilde{t}_{1} \tilde{t}_{1}(\%)$ & 1.8 & 1.5 & 41 & 65 & 0.01 \\
\hline$\widetilde{W}_{1} \widetilde{Z}_{2}(\%)$ & 43.8 & 45 & 26.5 & 18 & 16.7 \\
\hline$\widetilde{W}_{1} \overline{\tilde{W}}_{1}(\%)$ & 33.5 & 33 & 17.6 & 13 & 24.6 \\
\hline
\end{tabular}

assuming a pure phase space decay distribution. In this case, the peak in the signal distribution will merge with the background contribution from off-shell $Z$ decays in $W Z$ production, and much of the signal will be eliminated by the $Z$-veto cut. The phase space approximation will thus result in a significant overestimate of the signal in this case.

\section{FIVE CASE STUDIES}

In order to compare the five sets of cuts we have performed five case studies, each case being characterized by a qualitative feature of sparticle production mechanism or sparticle decay pattern as described below. These scenarios were first examined at the Fermilab Run II Workshop on SUSY/Higgs physics. The first four are realized within the MSUGRA framework, while in the fifth one non-universal soft SUSY breaking Higgs masses were chosen at the grand unified theory (GUT) scale to realize a "low $\mu$ scenario.", The model parameters for each of these cases are listed in Table III along with several sparticle masses and production cross sections for relevant SUSY production processes.

We note the following features of each case study point.

Case 1. The MSUGRA parameters for this point lie in the 
TABLE IV. SUSY $3 l$ signal (fb) for hard and soft cuts at the Fermilab Tevatron for Cases $1-5$ described in Sec. IV and for Cases A, B, and C corresponding to Figs. 6-8. The two columns for SC1 cuts refer to cross sections with and without the inclusion of decay matrix elements as discussed in the text.

\begin{tabular}{ccccccc}
\hline \hline Case & HC1 & SC1 (no ME) & SC1 (ME included) & SC2 & HC2 & SC3 \\
\hline$(1)$ & $3.3 \pm 0.2$ & $13.1 \pm 0.5$ & $13.1 \pm 0.5$ & $6.9 \pm 0.3$ & $3.5 \pm 0.2$ & $0.11 \pm 0.04$ \\
$(2)$ & $0.17 \pm 0.04$ & $1.6 \pm 0.1$ & $1.6 \pm 0.1$ & $0.8 \pm 0.1$ & $0.26 \pm 0.05$ & $0.26 \pm 0.06$ \\
$(3)$ & $0.30 \pm 0.10$ & $2.5 \pm 0.3$ & $2.5 \pm 0.3$ & $1.2 \pm 0.2$ & $0.41 \pm 0.1$ & $0.6 \pm 0.1$ \\
$(4)$ & $1.4 \pm 0.3$ & $3.6 \pm 0.5$ & $3.3 \pm 0.5$ & $2.1 \pm 0.4$ & $1.2 \pm 0.3$ & $0.07 \pm 0.07$ \\
$(5)$ & $0.3 \pm 0.1$ & $1.4 \pm 0.2$ & $1.2 \pm 0.2$ & $0.6 \pm 0.1$ & $0.33 \pm 0.1$ & $0.14 \pm 0.06$ \\
& & & & & \\
(A) & $7.4 \pm 0.4$ & $20.6 \pm 0.1$ & $19.6 \pm 0.6$ & $11.4 \pm 0.5$ & $8.0 \pm 0.4$ & $0.26 \pm 0.08$ \\
(B) & $0.16 \pm 0.05$ & $0.55 \pm 0.1$ & $0.6 \pm 0.1$ & $0.33 \pm 0.01$ & $0.19 \pm 0.05$ & $0.015 \pm 0.015$ \\
(C) & $0.16 \pm 0.02$ & $0.44 \pm 0.04$ & $0.25 \pm 0.02$ & $0.12 \pm 0.02$ & $0.10 \pm 0.02$ & $0.01 \pm 0.005$ \\
\hline \hline
\end{tabular}

cosmologically favored region of parameter space [11], giving rise to a reasonable relic density of neutralinos. The dominant production mechanisms at the Fermilab Tevatron are $\widetilde{W}_{1} \overline{\widetilde{W}}_{1}$ and $\widetilde{W}_{1} \widetilde{Z}_{2}$ production. For this case, $\widetilde{Z}_{2} \rightarrow l \widetilde{l}_{R}$ at $\sim 100 \%$, so a large rate for clean trilepton events is expected, and decay matrix element effects are unimportant.

Case 2. This parameter space point is selected to have a large value of $\tan \beta=35$ so that $\widetilde{W}_{1} \rightarrow \tilde{\tau}_{1} \nu_{\tau}$ and $\widetilde{Z}_{2} \rightarrow \tilde{\tau}_{1} \tau$ occur with a branching fraction of $\sim 100 \%$. The dominant production cross section is again $\widetilde{W}_{1} \bar{W}_{1}$ and $\widetilde{W}_{1} \widetilde{Z}_{2}$ production. Here, we anticipate that an inclusive trilepton signal can be more effectively extracted with relatively soft lepton $p_{T}$ cuts, since the detected leptons typically come from $\tau$ decays. Events containing a mixture of $(3-n) e$ s or $\mu \mathrm{s}$, together with $n \tau$ jets should also exist $(1 \leqslant n \leqslant 3)$.

Case 3. This parameter space point is also chosen with large $\tan \beta$, but the $A_{0}$ parameter was chosen so that relatively light $\tilde{t}_{1}, \tilde{b}_{1}$, and $\tilde{\tau}_{1}$ are generated. $\widetilde{W}_{1} \rightarrow \tilde{\tau}_{1} \nu_{\tau}$ and $\widetilde{Z}_{2}$ $\rightarrow \widetilde{\tau}_{1} \tau$ occur again at $\sim 100 \%$, but the masses of $\widetilde{W}_{1}$ and $\widetilde{Z}_{2}$ are about $20 \mathrm{GeV}$ smaller than in case 2, so that the trileptons should occur at about twice the rate as in case 2. Moreover, the rather large $\tilde{t}_{1} \overline{\tilde{t}}_{1}$ production cross section may yield an observable $\tilde{t}_{1}$ signal but we will not investigate this here. Once produced, $\widetilde{t}_{1} \rightarrow b \widetilde{W}_{1}$ with a branching fraction $\sim 100 \%$, but since $\widetilde{W}_{1} \rightarrow \tilde{\tau}_{1} \nu_{\tau}$, hard electrons or muons are not generated in the $\tilde{t}_{1}$ cascade decay. The cross section for all flavors of squarks and for gluinos is about $50 \%$ of the total cross section.

Case 4. This parameter choice leads to $\widetilde{W}_{1} \overline{\widetilde{W}}_{1}, \widetilde{W}_{1} \widetilde{Z}_{2}$ and $\tilde{t}_{1} \tilde{t}_{1}$ production as the main SUSY processes. It could yield a sample of high $p_{T}$ trilepton events. One may also search for $\tilde{t}_{1} \widetilde{t}_{1}$ production where $\widetilde{t}_{1} \rightarrow b \widetilde{W}_{1}$ with $\widetilde{W}_{1} \rightarrow l \nu_{l} \widetilde{Z}_{1}$, but this is beyond the scope of our study. Since charginos and neutralinos decay via three body modes, the decay matrix element effect may be important.

Case 5. This point was chosen to have rather large Higgs boson masses at the grand unified theory (GUT) scale, so that scalar universality is broken. The $\mu$ parameter is relatively small so that the lower lying charginos and neutralinos have a substantial higgsino component. In this case, $\widetilde{W}_{1} \bar{W}_{1}$, $\widetilde{W}_{1} \widetilde{Z}_{2}$ and $\widetilde{W}_{1} \widetilde{Z}_{3}$ all occur at large rates. $\widetilde{Z}_{2} \rightarrow e e \widetilde{Z}_{1}$ occurs with a $3 \%$ branching ratio, but $\widetilde{Z}_{3} \rightarrow \tilde{\tau}_{1} \tau$ at $\sim 100 \%$. This case may lead to clean, hard trileptons from $\widetilde{W}_{1} \widetilde{Z}_{2}$ production, but also contain a soft trilepton component from $\widetilde{W}_{1} \widetilde{Z}_{3}$ production. Decay matrix elements can again be important in this case.

\section{A. Observability of the SUSY signal}

The total cross section for production of all sparticles is shown in Table III. We also list here the percentage of cross section for various relevant sparticle production mechanisms. We see that while chargino and neutralino production dominate in cases 1,2 and 5, the production of squarks and gluinos (mainly stops and sbottoms) is important in cases 3 and 4.

The trilepton event cross sections after cuts for cases 1-5 introduced above are shown in Table IV for the five sets of cuts introduced in Table I. For the soft cuts the signal with SC1 exceeds SC2 because of the additional $\gamma^{*}$ and $W$-veto requirements. While these reduce the signal by $40-50 \%$, the corresponding reduction in the background is by a factor of 35 (mainly due to the reduction of the $W^{*} Z^{*}$ and $W^{*} \gamma^{*}$ backgrounds). Comparing with the BG rates from Table II, we see that with $2 \mathrm{fb}^{-1}$ none of the cases are observable at the $5 \sigma$ level using cuts $\mathrm{SC} 1$. Using cut set $\mathrm{SC} 2$, only case 1 is visible with $2 \mathrm{fb}^{-1}$ while cases 1,3 and 4 should be observable at $\mathrm{TeV} 33$. For the hard cuts, it is clear that the set $\mathrm{HC} 2$ performs better than the set $\mathrm{HC} 1$ : at $\mathrm{TeV} 33$, cases 1 and 4 are observable using $\mathrm{HC} 2$ in contrast to just case 1 via $\mathrm{HC} 1 .^{3}$ Notice also that with $\mathrm{SC} 3$ cuts none of the signal

\footnotetext{
${ }^{3}$ The reader may wonder that for cases 2 and 3 the signal with $\mathrm{HC} 2$ cuts is larger than that for $\mathrm{HC} 1$ cuts where all the requirements (except the jet veto) are milder. We have checked that in these cases bulk of the cross section comes from SUSY reactions (which may contain jets) other than $\widetilde{W}_{1} \widetilde{Z}_{2}$ production. In contrast, for case 1 , $83 \%(71 \%)$ of the signal with $\mathrm{HC} 1$ ( $\mathrm{HC} 2)$ cuts originates in the $\widetilde{W}_{1} \widetilde{Z}_{2}$ process.
} 
cases are observable with just $2 \mathrm{fb}^{-1}$ of integrated luminosity, and only case 3 is observable at TeV33. This is, of course, to be expected for the low $\tan \beta$ cases 1 and 4 , and for case 5 since neutralinos then decay to OS/SF dileptons. The point, however, is that although these cuts reduce the background very significantly, the corresponding loss of the signal is simply too large to make this strategy very promising even at TeV33. While these cuts offer the best signal to background ratio for cases 2 and 3, the significance $S / \sqrt{B}$ is worse than that for cuts SC2 in case 2, and in case 3 the two sets of cuts give about the same statistical significance. In some regions of parameter space cuts SC3 may thus be useful as a diagnostic once a signal has been found, but they do not increase the reach.

We conclude that over a large part of the parameter space, the inclusive soft cuts set SC2 appear to provide the best strategy for extracting the trilepton signal, since the dominant $W^{*} Z^{*}$ and $W^{*} \gamma^{*}$ backgrounds are largely reduced by the three extra cuts included here. It may, however, be that the cuts $\mathrm{HC} 2$ may provide a somewhat better reach for sufficiently large values of $m_{1 / 2}$ (and sometimes a better signal to background ratio).

Finally, to illustrate the impact of the matrix element, we also list in Table IV the cross section for the inclusive signal with soft cuts set SC1 but without the decay matrix elements included; i.e. as it was computed prior to the release of ISAJET 7.44. Of course, there is no change for the first three cases as three body decays of the chargino and neutralino were not important, but for cases 4 and 5, the inclusion of decay matrix elements decreases the predicted observable event rate by $10-15 \%$. We have also shown the signals for cases $\mathrm{A}, \mathrm{B}$ and $\mathrm{C}$ corresponding to MSUGRA parameters in Figs. 6, 7, and 8, respectively. Although the cross section is unobservably small except in case A, we have shown these as additional examples that enable the reader to gauge the importance of the matrix element effect for the evaluation of the reach. We see that as expected from Figs. 6 and 7, the matrix element has little effect for cases A and B. For case C, Fig. 8 would suggest that there should be a substantial increase in the cross section when matrix elements are included as these cause a substantial hardening of the lepton $p_{T}$ distributions; we see, however, that the cross section is substantially reduced. This is because, as we saw from Fig. 11, the matrix element causes the masses of the dileptons from $\widetilde{Z}_{2}$ decays to peak near to $M_{Z}$, so that these events are removed by the $Z$-veto. We conclude that at least for the soft cuts considered here, matrix element effects generally do not alter the signal cross section by more than $10-20 \%$ except for small regions of parameter space where a three-body decay is close to becoming a quasi-two-body decay. As pointed out in Ref. [14] this is not, however, the case for dilepton mass (and presumably other) distributions which we consider next.

\section{B. Dilepton invariant mass distributions}

It is known that the mass distribution of same flavor, opposite sign dileptons can provide important information about neutralino, and possibly also slepton, masses. This dis-
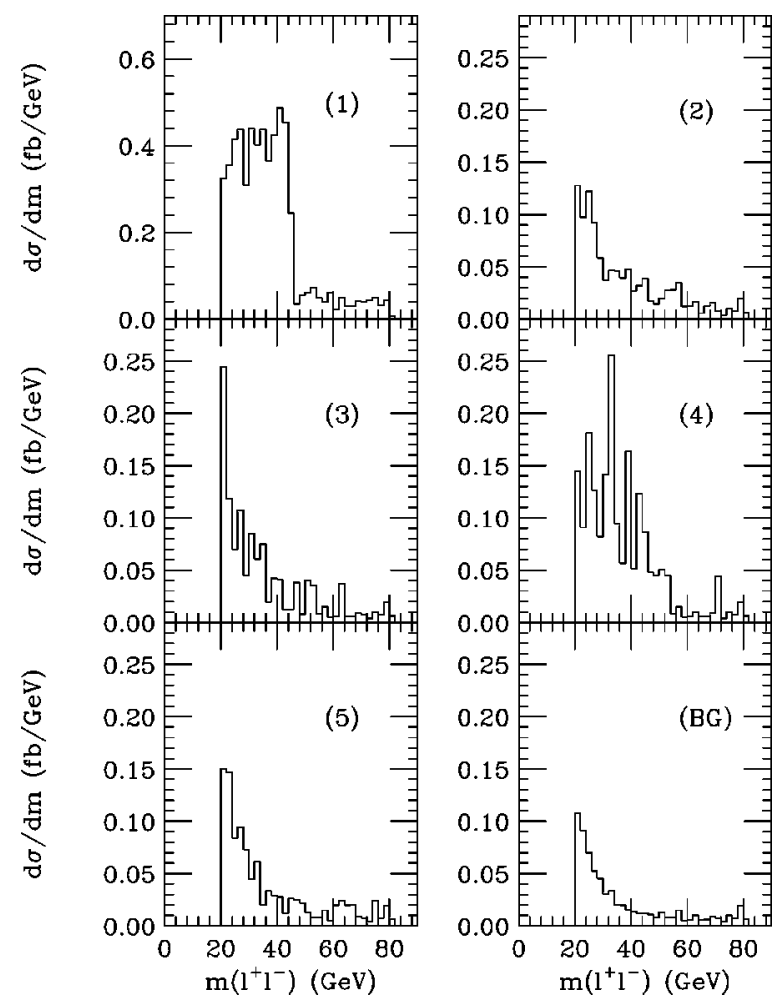

FIG. 12. Opposite sign same-flavor dilepton invariant mass distributions after cuts SC2 listed in Table I for the five case study points introduced in Sec. IV of the text. Each frame is labeled by the particular case number. The last frame labeled BG shows the sum of SM backgrounds from Table II. The background is included in the histogram for each case study. The cross section in each plot is greater than the value listed in Table IV since some events can have more than one plot entry.

tribution has recently been studied in Ref. [14] where interesting effects arising from the matrix element have been pointed out. The magnitude and nature of these effects is sensitive to underlying parameters, and so can (in some cases) provide an additional tool to obtain these. Here, we examine this distribution for the five cases that we have examined in detail, incorporating the inclusive soft cuts SC2 which for the five case studies yield the largest reach at luminosity upgrades of the Fermilab Tevatron. In several of the cases the leptons arise as secondaries from the decay of the parent tau that is produced via the decay of $\widetilde{Z}_{2}$. In this case, the dilepton mass distribution will be squeezed to lower mass values, and the sharp edge that directly provides information about sparticle masses is washed out. Nevertheless, we show these distributions as they illustrate what might be expected in such SUSY scenarios even though they only roughly yield information about neutralino or slepton masses.

In Fig. 12, we show the resulting dilepton mass distribution after the cuts SC2 and with the backgrounds included. The frames are labeled by the corresponding case number. The final histogram labeled BG shows the distribution from the various backgrounds shown in Table II with the same set of cuts. The rise at low $m(l \bar{l})$ is due to the tail of the $W^{*} \gamma^{*}$ background discussed earlier. This will obscure the determi- 
nation of the lower end point of this distribution. The sharp cut off at the high end is from the $Z$-veto cut - without this, the distribution has a huge peak at $m(l \bar{l})=M_{Z}$.

For case 1, the trilepton signal is large and a mass endpoint may be visible even at Run 2 where $\sim 14$ signal events are expected. In this case, $\widetilde{Z}_{2} \rightarrow l \bar{l} \widetilde{Z}_{1}$ via a real $\widetilde{l}_{R}$, so an edge is expected at

$$
m_{l \bar{l}}^{\max }=m_{\tilde{Z}_{2}} \sqrt{1-\frac{m_{\tilde{l}_{R}}^{2}}{m_{\tilde{Z}_{2}}^{2}}} \sqrt{1-\frac{m_{\tilde{Z}_{1}}^{2}}{m_{\tilde{l}_{R}}^{2}}} \simeq 45 \mathrm{GeV},
$$

and is clearly visible in the plot. With an integrated luminosity of $25 \mathrm{fb}^{-1}$ a moderately precise measurement of the end point should be possible.

For case 2, there should be a similar edge - but in the $m(\tau \bar{\tau})$ distribution-at $54.5 \mathrm{GeV}$. Dileptons from the subsequent $\tau$ leptonic decays should also respect this bound, but with a softened mass distribution. Indeed this appears to be the case when compared against the pure background distribution shown in the last frame: there is some signal enhancement beyond the SM expectation for dilepton masses ranging between about 25 and $50 \mathrm{GeV}$. We note, however, that when two of the three leptons are secondaries of taus from the neutralino in $\widetilde{W}_{1} \widetilde{Z}_{2}$ production, there is no reason to expect that the leptons from $\widetilde{Z}_{2}$ decay should have the same flavor. In fact in events where a tau each decays into $e$ and $\mu$, the same flavor pair necessarily comes in association with a lepton from elsewhere (here from chargino decay) and so should not be expected to respect this end point (except that the mass is small as the daugter lepton from tau will usually be soft). Furthermore, for case 2, production of charged sleptons and/or sneutrinos and, to a lesser extent heavier charginos and neutralinos, make significant contributions to the trilepton signal, frequently without any $\widetilde{Z}_{2}$ in the cascade. We thus expect no sharp edges and no real structure to the $m(l \bar{l})$ distribution for this case. Moreover, the event sample will be quite limited even with $25 \mathrm{fb}^{-1}$ of integrated luminosity since less than 20 signal events (and a comparable number of background events) make up the plot. Extraction of information on neutralino or slepton masses from this distribution appears to be very difficult. It might, however, be interesting to examine the possibility of constructing the mass edge using identified hadronically decaying $\tau$ 's; since this clearly depends on detector capabilities, we have not attempted to do so.

Case 3 should be very similar to case 2 as here, $\widetilde{Z}_{2}$ again essentially always decays via $\widetilde{Z}_{2} \rightarrow \tau \widetilde{\tau}_{1}$. In this case we expect $m(\tau \bar{\tau})$ should be bounded by $47 \mathrm{GeV}$. As in case 2 , the trilepton signal originates in many different SUSY sources. Aside from the usual $\widetilde{W}_{1} \widetilde{Z}_{2}$ production, third generation squarks and slepton/sneutrino production contribute significantly to this sample with smaller contributions from other SUSY reactions. It is interesting to see that the dilepton mass reconstruction again (mostly) respects the bound $m(l \bar{l})$

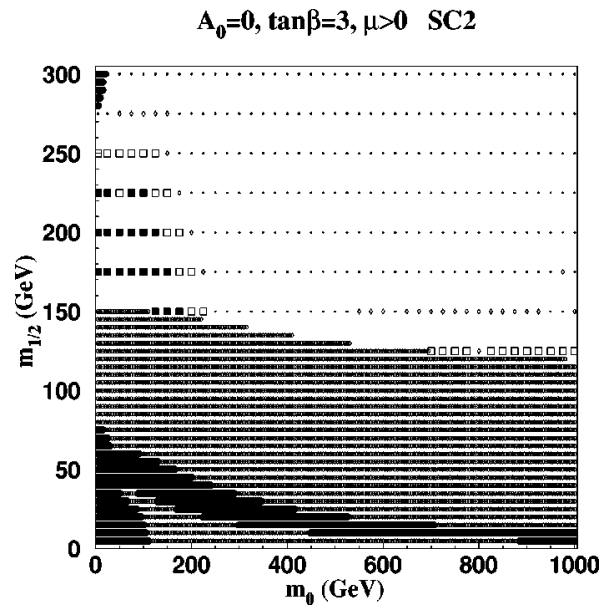

FIG. 13. The reach of the Fermilab Tevatron collider for MSUGRA model in the $m_{0}$ vs $m_{1 / 2}$ plane for $A_{0}=0, \tan \beta=3$ and $\mu>0$, using cuts SC2. The black shaded regions are theoretically excluded, while the gray areas are experimentally excluded by sparticle and Higgs boson searches at LEP2. The black squares denote points accessible to Tevatron experiments at the $5 \sigma$ level with just $2 \mathrm{fb}^{-1}$ of data, while open squares are accessible with $25 \mathrm{fb}^{-1}$. Points denoted by diamonds are accessible at the $3 \sigma$ level with 25 $\mathrm{fb}^{-1}$ of integrated luminosity.

$\leqslant m_{\tilde{Z}_{2}}-m_{\tilde{Z}_{1}}$; Cases 2 and 3 clearly show the care that must be exercised before inferring the origin of SUSY trilepton events even though the $m(l \bar{l})$ distribution clusters in a limited range. Extraction of precision mass information from this distribution again seems very difficult.

In case $4, \widetilde{Z}_{2} \rightarrow l \bar{l} \widetilde{Z}_{1}$ via virtual particles, so we expect $m(l \bar{l})$ to be bounded by $m_{\tilde{Z}_{2}}-m_{\tilde{Z}_{1}}=54 \mathrm{GeV}$. The mass edge, though not as sharp as in case 1 , is evident in the figure. It should, of course, be remembered that the signal cross section is just $2.1 \mathrm{fb}$ so that a substantial integrated luminosity will be needed to extract $m_{\tilde{Z}_{2}}-m_{\tilde{Z}_{1}}$ with any precision.

Finally, in case 5, dileptons can occur from $\widetilde{Z}_{2}$ via virtual sparticles or virtual $Z$, and also from $\widetilde{Z}_{3}$ decays via a real $\tilde{\tau}_{1}$. In the $m(l \bar{l})$ distribution shown in the figure we expect a mass edge at $m_{\tilde{Z}_{2}}-m_{\tilde{Z}_{1}}=44 \mathrm{GeV}$. The decay $\widetilde{Z}_{3} \rightarrow \tau \tilde{\tau}_{1}$ will likewise have a $m(\tau \bar{\tau})$ edge at $39 \mathrm{GeV}$ with a correspondingly softer dilepton mass distribution. While there is a slight dip in the distribution at $\sim 55 \mathrm{GeV}$, a clear mass edge does not appear evident. Note that with $25 \mathrm{fb}^{-1}$ of integrated luminosity, only about 15 signal events will be used to create this plot, so the statistical sample will be very limited. This scenario is further complicated by the fact that both $\widetilde{W}_{1} \widetilde{Z}_{2}$ and $\widetilde{W}_{1} \widetilde{Z}_{3}$ production is substantial. Extraction of masses, though possible, in principle, appears to be difficult. It would be interesting to examine whether the detection of a signal with taus identified via their hadronic decays would make it possible to identify the additional presence of $\widetilde{Z}_{3}$ in the SUSY event sample. 


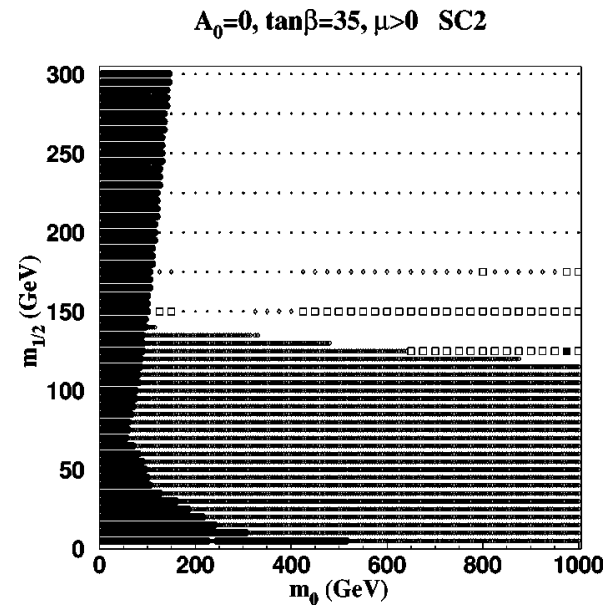

FIG. 14. Same as Fig. 13, except for $\tan \beta=35$.

\section{REACH OF FERMILAB TEVATRON FOR MSUGRA VIA THE INCLUSIVE ISOLATED TRILEPTON CHANNEL}

In this section, we use the augmented cut sets SC2 and HC2 (which effectively removed the bulk of the new backgrounds evaluated here) to calculate the SUSY trilepton signal, and thereby determine the reach of Fermilab Tevatron upgrades in the parameter space of the MSUGRA model. We present results for $\mu>0$, since much of the parameter space for $\mu<0$ and low $\tan \beta$ is ruled out by the recent LEP2 bound on the mass of the Higgs boson. For large values of $\tan \beta$, the reach plots become similar regardless of the sign of $\mu$. We note that values of $\mu<0$ also seem to be disfavored from comparing model predictions for $b \rightarrow s \gamma$ decay rates [21] to experimental results from CLEO [22] and ALEPH [23].

In earlier studies $[5,6,8]$, reach results were presented for the Fermilab Tevatron for the low $\tan \beta=2$ value. All the observable regions for $\tan \beta=2$ are now ruled out by the LEP2 result that $m_{h}>95 \mathrm{GeV} .{ }^{4}$ Increasing $\tan \beta$ by just one unit, to $\tan \beta=3$, typically raises the value of $m_{h}$ by $10 \mathrm{GeV}$, placing it beyond the current reach of LEP2 Higgs searches.

To illustrate the reach of the Tevatron for low $\tan \beta$ values not accessible at LEP2, we evaluate the observability of the SUSY trilepton signal for $\tan \beta=3$ and show our results in Fig. 13 for $\tan \beta=3$ in the $m_{0}$ vs $m_{1 / 2}$ plane, for $A_{0}=0$ and $m_{t}=175 \mathrm{GeV}$. The black regions are excluded by theoretical constraints: either electroweak symmetry is not correctly broken, or the lightest SUSY particle is charged or colored. The gray regions are excluded by constraints from LEP2 that $m_{\tilde{W}_{1}}>95 \mathrm{GeV}$ [3]. We have scanned the points on a $(25 \mathrm{GeV} \times 25 \mathrm{GeV})$ grid on this plane to see whether the SUSY signal is observable above background at the $\geqslant 5 \sigma$ level. At parameter space points marked with a black square the signal cross section after cuts exceeds $3.62 \mathrm{fb}$, and so should be observable at Tevatron Run II with SC2 cuts,

\footnotetext{
${ }^{4}$ The LEP2 bound [24] is obtained for the Higgs boson of the standard model. It should, however, also be applicable to $h$ since for low values of $\tan \beta, h$ is essentially the SM Higgs boson.
}

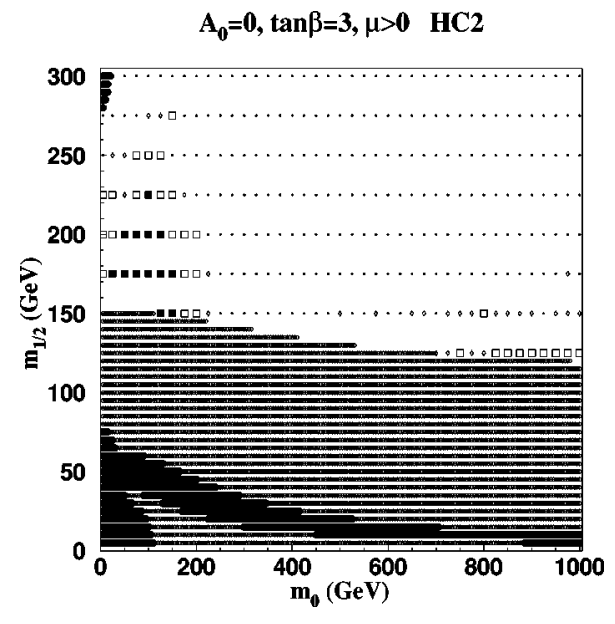

FIG. 15. Same as Fig. 13, except for cuts HC2.

assuming an integrated luminosity of $2 \mathrm{fb}^{-1}$. At points marked by an open square the cross section exceeds $1.02 \mathrm{fb}$, and hence are considered to give a detectable signal at TeV33 at $5 \sigma$ level, assuming $25 \mathrm{fb}^{-1}$ of integrated luminosity. Also shown as diamonds are points accessible at the $3 \sigma$ level at TeV33. While this is not a discovery limit, it gives the reader an idea of the parameter range where tantalizing hints of SUSY might be possible. Points where the signal is undetectable even at this level with $25 \mathrm{fb}^{-1}$ of integrated luminosity are denoted by dots.

In this case, the Tevatron reach with just $2 \mathrm{fb}^{-1}$ extends to $m_{1 / 2}=225 \mathrm{GeV}$. The reach of the Fermilab Tevatron with $25 \mathrm{fb}^{-1}$ typically extends to $m_{1 / 2}=250 \mathrm{GeV}$, corresponding to $m_{\tilde{g}} \sim 660 \mathrm{GeV}$ and $m_{\widetilde{W}_{1}} \sim 200 \mathrm{GeV}$. Coincidentally, this is the same as the $\tan \beta=2$ reach $[6,7]$ quoted for the clean $3 l$ channel (but neglecting the $W^{*} Z^{*}$ and $W^{*} \gamma^{*}$ backgrounds). For $\tan \beta=3$, the reach in $m_{0}$ is rather limited, and cuts off sharply at $m_{0}=200-225 \mathrm{GeV}$, where the $\widetilde{Z}_{2}$ leptonic branching fraction severely decreases due to interference effects. Some reach for TeV33 is recovered at very large $m_{0}>500 \mathrm{GeV}$ for small $m_{1 / 2}$ where the $Z$ pole begins to dominate the $\widetilde{Z}_{2}$ leptonic branching fraction.

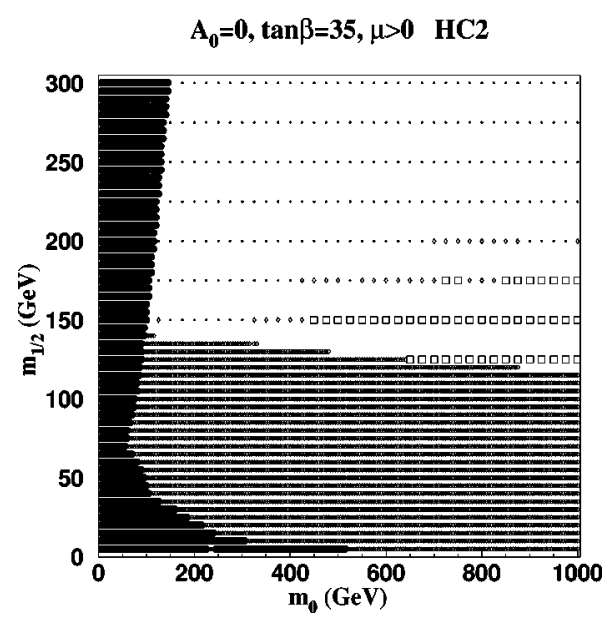

FIG. 16. Same as Fig. 15, except for $\tan \beta=35$. 
In Fig. 14, we show the corresponding reach of the Fermilab Tevatron using SC2 for a large value of $\tan \beta=35$. In this case, for low values of $m_{0}, \widetilde{Z}_{2}$ and $\widetilde{W}_{1}$ decay dominantly to $\tau$ leptons due to the dominance of decays mediated by (real or virtual) $\tilde{\tau}$ 's. None of the parameter space points examined yields an observable signal at the Fermilab Tevatron with just $2 \mathrm{fb}^{-1}$. A reach significantly beyond the current LEP2 limits is possible if $25 \mathrm{fb}^{-1}$ of data is accumulated, especially at large $m_{0}$. In addition, many of these same points plus others are accessible via the $\boldsymbol{E}_{T}+$ jets and $l l \tau$ or $l \tau \tau$ signals discussed in Ref. [7].

Similar reach plots $^{5}$ are shown for the same parameter planes using the cuts HC2 in Figs. 15 and 16. Qualitatively, much of the reach is similar, although HC2 work better in Fig. 15 around $m_{0} \sim 200 \mathrm{GeV}$ and large $m_{1 / 2}$ (where the signal leptons are expected to be hard), whereas SC2 work better at very low $m_{0}$ (where the mass difference between the sleptons and $\widetilde{Z}_{1}$ is small). In Fig. 16, we see that SC2 work better at low $m_{0}$ where the trileptons occur dominantly from cascade decays involving $\tau$ leptons. For large $m_{0}$, the $\widetilde{Z}_{2}$ decays are dominated by the $Z$ exchange graph. In this case, HC2 work slightly better at very large $m_{1 / 2}$. Further optimization of cuts is possible as already noted.

\section{SUMMARY AND CONCLUDING REMARKS}

Within the MSUGRA model (or any framework with a common gaugino mass at a high scale $\sim M_{G U T}$ ), the LEP2 bound $m_{\tilde{W}_{1}} \geqslant 95 \mathrm{GeV}$ translates to $m_{g} \gtrsim 300 \mathrm{GeV}$. In this case, electroweak production of charginos and neutralinos is the dominant sparticle production process at the Tevatron, and the trilepton signal from $\widetilde{W}_{1} \widetilde{Z}_{2}$ production assumes even greater importance than in the past. It has recently been emphasized that the SUSY reach via this channel can be increased by softening the $p_{T}$ requirements on the leptons, and further, by not imposing a jet veto on these events. In contrast to these developments that enhance the reach relative to previous projections, it has also been pointed out that $W Z^{*}$ $\rightarrow 3 l$ production, which had been omitted in previous analyses, is a major source of SM background to the trilepton signal; the inclusion of this new background of course reduces the reach. Moreover, as the $m(l \bar{l})$ spectrum from SUSY events (see Figs. 9-11) extends to very low values, SM background from $W \gamma^{*}$ background (which had never been evaluated before) needed to be incorporated. Finally, previous studies of the trilepton signal ignored the effect of the chargino and neutralino decay matrix elements in the evaluation of the energy and angular distributions of the leptons. While these do not alter total rates, the rates after experimental cuts can be changed. Recently, the tree level decay matrix elements have been included in the event generator ISAJET which is often used to compute the SUSY signal. In view of these developments and also the impor-

${ }^{5}$ We have explicitly checked that even for the $\tan \beta=35$ case at TeV33 there is very little reach via SC3 cuts. tance of the SUSY trilepton signal, we felt a reassessment of the signal was warranted. An improved calculation of the Tevatron reach via this channel is the main subject of this paper.

For the soft inclusive cuts used here, incorporation of the matrix elements, for the most part, changes the signal cross sections by less than $\pm 15 \%$ so that the conclusions about signal levels from other studies where these are ignored should be qualitatively correct. This is not to say matrix element effects are always unimportant. First, there are regions of parameter space where the matrix elements do qualitatively change the distributions (e.g. case C in Sec. III), and hence, signal cross sections. More importantly, matrix element effects can considerably distort the distribution of same flavor opposite sign dileptons in SUSY trilepton events from which important information about neutralino, and perhaps also slepton, masses may be obtained. This issue may be even more important for SUSY studies at $e^{+} e^{-}$linear colliders or at the LHC where, because of the clean environment and/or the large rates, the extraction of sparticle masses is an integral part of the SUSY program.

Turning to our main purpose, we have shown that augmenting previously proposed soft or hard cuts with additional cuts designed to reduce $W \gamma^{*}$ and $W Z^{*}$ backgrounds can still lead to substantial regions of parameter space where the trilepton signal should be observable at Fermilab Tevatron upgrades. Our updated projection of the Fermilab Tevatron reach via the trilepton channel is summarized in Figs. $13,14,15$, and 16 for both low and high $\tan \beta$, as well as hard and soft lepton $p_{T}$ cuts. Soft cuts perform slightly better for the anticipated luminosity of Run II. However, with 25 $\mathrm{fb}^{-1}$ of data, the hard cuts can extend the reach towards larger values of the gaugino mass parameter $m_{1 / 2}$, for some values of the scalar mass parameter $m_{0}$. It is noteworthy that a $5 \sigma$ signal at Run II requires a signal to background ratio of at least 3.5 to 1 (5 to 1 for the hard cuts). In contrast, at TeV33 and with soft cuts, signal and background are about equal at the $5 \sigma$ discovery limit: a good knowledge of the background normalization is then very important.

Overall we find that, while the reach is limited in that there are large ranges of parameters where experiments at the Fermilab Tevatron may see no signal in this channel even if $m_{\widetilde{W}_{1}}$ is just beyond the LEP2 bound, experiments at Run II should be able to probe significant parameter ranges not accessible at LEP if $\tan \beta$ is small. For $\tan \beta=35$ (or larger) there is very little signal in this channel at Run II, although some ranges of parameters become accessible with $25 \mathrm{fb}^{-1}$ of integrated luminosity. This underscores the importance of other channels for SUSY detection, particularly when $\tan \beta$ is large. The standard $\boldsymbol{E}_{T}$, the $\mathbb{E}_{T}$ plus tagged $b$, the $l l \tau$ and $l \tau \tau$ (where $\tau$ is identified via its hadronic decays) channels are especially important $[7,10]$ in this regard. Since we do not know what nature has in store for us, we reiterate the need to develop techniques to efficiently tag $\tau$ leptons via their hadronic decays to ensure that new physics signals (not necessarily from supersymmetry) do not evade detection at luminosity upgrades of the Fermilab Tevatron. The develop- 
ment of tau triggers does not appear to be as essential at this point.

Before closing, we mention that it may be possible to further optimize the cuts once a SUSY signal has been detected. For instance, if data indicate that the upper end point of the dilepton mass distribution happens to be well below $M_{Z}$, it may be possible to significantly reduce the dominant $W Z^{*}$ background (thereby increasing the significance of the signal) by widening the $Z$-veto window. The point, of course, is that once we have a qualitative picture of the signal, further optimization is likely to be possible.

Note added: After completion of this work, a revised version of Ref. [9] appeared which also presented a calculation of the $W^{*} Z^{*}$ and $W^{*} \gamma^{*}$ backgrounds. Our background calculations agree with those of Ref. [9] when the entire phase space is integrated over. Updated work by Matchev and Pierce [25] also includes these backgrounds. Reference [9] also includes (sub-dominant) background contributions from off-shell vector boson decays to $\tau$ s that we have neglected in our analysis. The conclusions for the reach obtained in both these studies appear to be in good qualitative agreement with ours.

\section{ACKNOWLEDGMENTS}

We thank C. Kao and V. Barger for discussions and collaboration on the Fermilab Run II SUGRA subgroup report, which led to this project. We also thank P. Mercadante and $\mathrm{Y}$. Wang for numerical checks on some of our results, and $\mathrm{K}$. Matchev and D. Pierce for discussions concerning the $W Z^{*}$ background. We are grateful to C. Kao and K. Matchev for comparisons that made us realize the importance of the contributions to the trilepton background from $W Z^{*}$ and $W \gamma^{*}$ production with the $W$ boson far off-shell: contributions from $M_{W^{*}}<70 \mathrm{GeV}$, which had been ignored in an earlier version of this manuscript, significantly increase the background for low values of $m_{l l}$ and led us to increase the dilepton mass cut for the SC2 case to $m_{l l} \geqslant 20 \mathrm{GeV}$. The work of M.D. was supported by FAPESP (Brazil). This work was supported in part by the U. S. Department of Energy under contract number DE-FG02-97ER41022, DE-AC02-98CH10886, and DE-FG-03-94ER40833.
[1] A. Chamseddine, R. Arnowitt, and P. Nath, Phys. Rev. Lett. 49, 970 (1982); R. Barbieri, S. Ferrara, and C. Savoy, Phys. Lett. 119B, 343 (1982); L. J. Hall, J. Lykken, and S. Weinberg, Phys. Rev. D 27, 2359 (1983); for a review, see H. P. Nilles, Phys. Rep. 110, 1 (1984).

[2] F. Paige, S. Protopopescu, H. Baer, and X. Tata, hep-ph/9804321 (1998).

[3] See e.g. E. Lancon, ALEPH Status report talk given at CERN LEPC meeting, November, 1998.

[4] H. Baer, K. Hagiwara, and X. Tata, Phys. Rev. Lett. 57, 294 (1986); Phys. Rev. D 35, 1598 (1987); R. Arnowitt and P. Nath, Mod. Phys. Lett. A 2, 331 (1987); R. Barbieri, F. Caravaglios, M. Frigeni, and M. Mangano, Nucl. Phys. B367, 28 (1991); H. Baer and X. Tata, Phys. Rev. D 47, 2739 (1993); J. Lopez, D. Nanopoulos, X. Wang, and A. Zichichi, ibid. 48, 2062 (1993); H. Baer, C. Kao, and X. Tata, ibid. 48, 5175 (1993); S. Mrenna, G. Kane, G. D. Kribs, and J. D. Wells, ibid. 53, 1168 (1996).

[5] H. Baer, C. H. Chen, C. Kao, and X. Tata, Phys. Rev. D 52, 1565 (1995).

[6] H. Baer, C. H. Chen, F. Paige, and X. Tata, Phys. Rev. D 54, 5866 (1996).

[7] H. Baer, C. H. Chen, M. Drees, F. Paige, and X. Tata, Phys. Rev. Lett. 79, 986 (1997); Phys. Rev. D 58, 075008 (1998).

[8] V. Barger, C. Kao, and T. Li, Phys. Lett. B 433, 328 (1998).

[9] V. Barger and C. Kao, Phys. Rev. D 60, 115015 (1999).

[10] J. Lykken and K. Matchev, Phys. Rev. D 61, 015001 (2000); K. Matchev and D. Pierce, ibid. 60, 075004 (1999).

[11] See, for instance, M. Drees and M. Nojiri, Phys. Rev. D 47,
376 (1993); H. Baer and M. Brhlik, ibid. 53, 597 (1996); 57, 567 (1998); V. Barger and C. Kao, ibid. 57, 3131 (1998); J. Ellis, T. Falk, K. Olive, and M. Srednicki, hep-ph/9905481 (1999).

[12] See, Baer and Tata, Ref. [4].

[13] Report of the MSUGRA Group in Proceedings of the Fermilab Tevatron Run II Workshop on SUSY/Higgs (unpublished).

[14] M. Nojiri and Y. Yamada, Phys. Rev. D 60, 015006 (1999).

[15] CDF Collaboration, F. Abe et al., Phys. Rev. Lett. 76, 4307 (1996).

[16] D0 Collaboration, S. Abachi et al., Phys. Rev. Lett. 76, 2228 (1996).

[17] See Mrenna et al. [4].

[18] Madgraph, by T. Stelzer and W. F. Long, Comput. Phys. Commun. 81, 357 (1994); HELAS, by H. Murayama, I. Watanabe, and K. Hagiwara, KEK-91-11 (1992).

[19] J. M. Campbell and R. K. Ellis, Phys. Rev. D 60, 113006 (1999).

[20] B. K. Bullock, K. Hagiwara, and A. D. Martin, Nucl. Phys. B395, 499 (1993).

[21] H. Baer and M. Brhlik, Phys. Rev. D 55, 3201 (1997); H. Baer, M. Brhlik, D. Castaño, and X. Tata, ibid. 58, 015007 (1998).

[22] CLEO Collaboration, M. S. Alam et al., Phys. Rev. Lett. 74, 2885 (1995).

[23] ALEPH Collaboration, R. Barate et al., Phys. Lett. B 429, 169 (1998).

[24] Report from the L3 Collaboration at the LEP Experiments Committee meeting, CERN, 1998.

[25] K. Matchev and D. Pierce, Phys. Lett. B 467, 225 (1999). 